\title{
NEAR-LINEAR DYNAMICS IN KDV WITH PERIODIC BOUNDARY CONDITIONS
}

\author{
M. B. ERDOĞAN, N. TZIRAKIS, AND V. ZHARNITSKY
}

\begin{abstract}
Near linear evolution in Korteweg de Vries (KdV) equation with periodic boundary conditions is established under the assumption of high frequency initial data. This result is obtained by the method of normal form reduction.
\end{abstract}

\section{INTRODUCTION}

This articles investigates the behavior of a class of solutions with high frequency initial data of Korteweg de Vries (KdV) equation,

$$
v_{t}=6 v v_{x}-v_{x x x}
$$

with periodic boundary conditions $v(x+2 \pi)=v(x)$. We show that, see Theorem 2.1 below, these solutions evolve near linearly, (i.e. like solutions of $v_{t}=-v_{x x x}$ ) for large times.

On the real line, near linear behavior for dispersive PDEs, such as nonlinear Schrödinger equation, nonlinear Klein-Gordon equations, KdV, etc., could be expected. Indeed, in that case high frequency solutions will disperse over a large subset of the real line weakening the nonlinearity. For example, under some conditions, one can extend the $L^{1}-L^{\infty}$ dispersive estimates for the linear Schrödinger equation to NLS, see e.g. [5]. In the focusing case, linear evolution could be destroyed by the mass concentration phenomenon as it leads to larger nonlinear effects. However, such concentration cannot occur in the case of mass subcritical nonlinearity. In short, there are two major reasons why on the real line, the evolution of high frequency solutions in the mass subcritical NLS case should be near linear: dispersive decay and absence of collapse.

For the $\mathrm{KdV}$ on the torus or a circle (periodic boundary conditions), the linear solution is periodic in space and time and, thus, one does not have dispersive decay. It is also generally believed that the solutions of $\mathrm{KdV}$ on the torus will not be approximated by the linear solutions as time goes to infinity. Therefore, it is somewhat surprising that, as we show in this paper, the evolution is still near linear on a finite but large time scale. One can argue that a hint towards this behavior comes from Bourgain's discovery

Date: November 13, 2018.

The authors were partially supported by NSF grants DMS-0600101 (B. E.), DMS0901222 (N. T.), and DMS-0807897 (V. Z.). 
of Strichartz estimates for periodic case [1, 2]. In some way, the effect of dispersion for the periodic problem can be interpreted as averaging of the nonlinearity over high frequencies.

On the torus there are other reasons, such as resonances, which could prevent linear behavior. For NLS, see [4], such resonances cause faster phase rotation while the behavior is still linear.

Our results are also motivated by the scattering problems for dispersive PDEs. On the real line there are many results on scattering, which show that nonlinear solutions tend to the linear ones as time goes to infinity. On the torus, however, one does not expect scattering. For example, the absence of scattering was proved rigorously for the cubic NLS on the two dimensional torus in [3]. Our statement is different since we only claim linear behavior for large but finite time scale for a special class of high frequency solutions. On the other hand, our near linear solutions provide some scattering like behavior.

Although $\mathrm{KdV}$ with periodic boundary conditions is completely integrable, our methods do not rely on integrability. We only use the conservation of momentum, energy, and Hamiltonian. An interesting question is whether integrability structure can be used to obtain more precise results on near linear evolution and on a larger time scale.

Our work also suggests a new mechanism of formation of the so-called rogue waves. Rogue waves (also called freak and giant waves) correspond to large-amplitude waves appearing on the sea surface "from nowhere". In the scientific literature, the following amplitude criterion for the rogue wave is usually used: its height should exceed the significant wave height (on the sea surface) by about a factor of two [7].

There is a vast literature on rogue waves, see e.g. the survey paper [7] and references therein, and many explanations have been proposed. Some scenarios involve

- probabilistic approach - rogue waves are considered as rare events in the framework of Rayleigh statistics

- linear mechanism - dispersion enhancement (spatio-temporal focusing)

- nonlinear mechanisms - in approximating models (e.g. NLS or $\mathrm{KdV}$ ), for some special initial data large amplitude waves can be created.

Linear mechanism of rogue wave formation is simpler since there are various solutions leading to large amplitudes, while nonlinear mechanism requires very special initial data. On the other hand, linear approximations are valid in the small amplitude limit which is restrictive. This article shows that for $\mathrm{KdV}$ the linear and nonlinear mechanisms can be combined into one since we describe a large subset of initial data for which the solutions of KdV equation behave near linearly. 
Regarding the boundary conditions, our choice of periodic boundary conditions is not the most realistic one but appropriate for a model problem. Indeed, while the sea surface is not periodic, one observes more or less similar pattern over large areas.

We finally mention that it would be best to observe near-linear dynamics for the full water wave problem, however, it is a considerably harder problem which will be addressed in future work. We also limit our study to the one dimensional problem.

\section{Main Results}

We consider $\mathrm{KdV}$ equation

$$
v_{t}=6 v v_{x}-v_{x x x}
$$

with periodic boundary conditions $v(x+2 \pi)=v(x)$ and we assume $v \in H^{1}\left(S^{1}\right)$. In this case, $\mathrm{KdV}$ is well-posed [9] and can be written in Hamiltonian form

$$
v_{t}=\frac{d}{d x} \frac{\partial H}{\partial v}
$$

where the Hamiltonian is given by

$$
H(v)=\int_{-\pi}^{\pi}\left(\frac{1}{2} v_{x}^{2}+v^{3}\right) d x
$$

and $\frac{\partial H}{\partial v}$ denotes $L^{2}$-gradient of $H$, representing the Fréchet derivative of $H$ with respect to the standard scalar product on $L^{2}$. We also need to consider linear part of $\mathrm{KdV}$

$$
v_{t}+v_{x x x}=0,
$$

with the solution given by

$$
v(x, t)=e^{L t} v(x, 0)
$$

where $L=-\partial_{x x x}$.

While KdV possesses infinitely many conserved quantities, we use the first three: the above Hamiltonian, linear momentum

$$
P=\int_{-\pi}^{\pi} v(x) d x
$$

and kinetic energy

$$
K=\int_{-\pi}^{\pi} v^{2}(x) d x
$$

Theorem 2.1. Assume without loss of generality that $P=0$ and

$$
\|v(\cdot, 0)\|_{H^{1}} \leq C \varepsilon^{-1},\|v(\cdot, 0)\|_{H^{-1 / 2}} \leq C \varepsilon^{1 / 2} .
$$

\footnotetext{
${ }^{1}$ One can reduce the case $P \neq 0$ to the zero momentum case $P=0$ by a simple transformation.
} 
for some $C>0$ and for sufficiently small $\varepsilon>0$. Then for any $t \lesssim \varepsilon^{-\frac{1}{2}+}$

$$
\left\|v(\cdot, t)-e^{L t} v(\cdot, 0)\right\|_{L^{2}} \lesssim\langle t\rangle \varepsilon^{\frac{1}{2}-}
$$

where the implicit constant depends only on $C$ but not on $\varepsilon$.

This Theorem follows from Theorem 2.2 below, which is proved by applying near-identical canonical transformations, so that the new Hamiltonian flow is close to the linear one. This implies that the original Hamiltonian flow is also close to the linear one.

Remark 2.1. Note that since the Hamiltonian (2) and the kinetic energy (41) are conserved quantities, the bounds (5) imply $|H(v(t))| \lesssim \varepsilon^{-2}$. This immediately implies a uniform bound in time $\|v\|_{H^{1}} \lesssim \epsilon^{-1}$.

To prove our theorem we first apply the following transformation [6], which is a weighted modification of Fourier transform

$$
v(x)=\sum_{n \neq 0} \sqrt{|n|} e^{i n x} u(n),
$$

where $n \in \mathbb{Z} \backslash\{0\}$ and $u(n)$ is a bi-infinite sequence of complex numbers. Since, $v(x)$ is real,

$$
u(-n)=\overline{u(n)}
$$

In these new variables the Hamiltonian takes the form 2

$$
\begin{aligned}
H & =i \sum_{n>0} n^{3} u(n) u(-n)+i \sum_{n_{1}+n_{2}+n_{3}=0} \sqrt{n_{1} n_{2} n_{3}} u\left(n_{1}\right) u\left(n_{2}\right) u\left(n_{3}\right) \\
& =: \Lambda_{2}+H_{3},
\end{aligned}
$$

where $\Lambda_{2}$ and $H_{3}$ are the quadratic and cubic parts of the Hamiltonian. Equivalently, in order to deal with the summation over all $n \neq 0$, we can write

$$
\Lambda_{2}=\frac{i}{2} \sum_{n \neq 0} n^{3} \sigma(n) u(n) u(-n),
$$

where $\sigma(n):=\operatorname{sgn}(n)$. In this formulation $u(m)$ and $u(-m)$, with $m=$ $1,2, \ldots$ are conjugated canonical variables with the standard symplectic structure, so that the Hamiltonian equations take the usual form

$$
\begin{aligned}
\frac{d u(m)}{d t} & =\frac{\partial H}{\partial u(-m)} \\
\frac{d u(-m)}{d t} & =-\frac{\partial H}{\partial u(m)},
\end{aligned}
$$

where $m>0$. We also write these equations in a more compact form

$$
\frac{d u(m)}{d t}=\sigma(m) \frac{\partial H}{\partial u(-m)}, \text { where } m \neq 0 \text {. }
$$

\footnotetext{
${ }^{2}$ Below we will omit the absolute value sign $|*|$ under the square root. It will be implicitly implied for the rest of the paper.
} 
It is straightforward to verify that these are the correct equations, by applying the change of variable (마) directly to $\mathrm{KdV}$.

Now, we introduce a subset of $l^{2}$

$$
X_{\varepsilon}^{\rho}=\left\{u \in l^{2}: u(0)=0, u(-n)=\bar{u}(n),\|u\|_{l^{2}} \leq \rho \sqrt{\varepsilon},\|u\|_{l_{3 / 2}^{2}} \leq \frac{\rho}{\varepsilon}\right\},
$$

where

$$
\|u\|_{\ell_{s}^{2}}^{2}=\sum_{k}|k|^{2 s}|u(k)|^{2} .
$$

We will also need the norm

$$
\|u\|_{\ell_{s}^{p}}^{p}=\sum_{k}|k|^{p s}|u(k)|^{p} .
$$

Note that the hypothesis of Theorem 2.1 is equivalent to $u \in X_{\varepsilon}^{\rho}$ initially in time for some $\rho>0$. By Remark 2.1, for any $t>0,\|u(\cdot, t)\|_{l_{3 / 2}^{2}} \lesssim \varepsilon^{-1}$. For the initial data in this subset we prove that the evolution is near linear.

Theorem 2.2. Let $\rho>0$ be fixed. Assume $u(\cdot, 0) \in X_{\varepsilon}^{\rho}$ for sufficiently small $\varepsilon>0$. Then for any $t \lesssim \varepsilon^{-\frac{1}{2}+}, u(\cdot, t) \in X_{\varepsilon}^{2 \rho}$ and

$$
\left\|u(n, t)-e^{i n^{3} t} u(n, 0)\right\|_{l_{1 / 2}^{2}(n)} \lesssim\langle t\rangle \varepsilon^{\frac{1}{2}-} .
$$

Theorem 2.1 immediately follows from this one by applying the relation $u(n)=\hat{v}(n) / \sqrt{|n|}$. To prove Theorem 2.2, we apply two canonical transformations $\Phi_{F_{1}}^{1}, \Phi_{F_{2}}^{1}$, see the next section, so that $u=u(q)=\Phi_{F_{1}}^{1} \circ \Phi_{F_{2}}^{1}(q)$. The new Hamiltonian is given by

$$
H(q)=H(u(q))=\Lambda_{2}(q)+R(q),
$$

where $R$ stands for the reminder terms, and the equations take the form

$$
\dot{q}(n)=i n^{3} q(n)+E(q)(n),
$$

where

$$
E(q)(n)=\frac{\partial}{\partial q(-n)} R, \quad n>0 .
$$

The transformation is near-identical in the following sense:

Proposition 2.1. If $u \in X_{\varepsilon}^{\rho}$ or $q \in X_{\varepsilon}^{\rho}$, then

$$
\|u(q)-q\|_{l_{s}^{2}} \lesssim \varepsilon^{1-s}
$$

where $s \in[0,3 / 2]$ and the implicit constant depends on $\rho, s$ but not on $\varepsilon$. In particular, for sufficiently small $\varepsilon$, if $q \in X_{\varepsilon}^{\rho}$, then $u(q) \in X_{\varepsilon}^{2 \rho}$ and vice versa.

The estimate for the error term is given by

Proposition 2.2. If $q \in X_{\varepsilon}^{\rho}$ then the error term satisfies

$$
\|E(q)\|_{l_{s}^{2}} \lesssim \varepsilon^{1-s-}
$$

where $s \in[0,1 / 2]$ and the implicit constant depends on $\rho, s$ but not on $\varepsilon$. 
Proof of Theorem 2.2. The proof follows easily from Propositions 2.1 and 2.2. Indeed, multiplying (9) with the integrating factor $e^{-i n^{3} t}$ and integrating from 0 to $t$, we obtain

$$
q(n, t) e^{-i n^{3} t}-q(n, 0)=\int_{0}^{t} e^{-i n^{3} \tau} E(q)(n) d \tau .
$$

Next, by taking the $\ell_{s}^{2}$ norm after multiplying both sides with $e^{i n^{3} t}$, we obtain

$$
\left\|q(n, t)-e^{i n^{3} t} q(n, 0)\right\|_{l_{s}^{2}}=\left\|\int_{0}^{t} e^{i n^{3}(t-\tau)} E(q)(n) d \tau\right\|_{l_{s}^{2}} \leq|t|\|E\|_{l_{s}^{2}} \lesssim|t| \varepsilon^{1-s-}
$$

for $s \in[0,1 / 2]$ and $t \lesssim \varepsilon^{-\frac{1}{2}+}$.

Then, using the triangle inequality, we estimate, for $s \in[0,1 / 2]$,

$$
\begin{aligned}
& \left\|u(n, t)-e^{i n^{3} t} u(n, 0)\right\|_{l_{s}^{2}} \leq \\
& \leq\|u(n, t)-q(n, t)\|_{l_{s}^{2}}+\left\|q(n, t)-e^{i n^{3} t} q(n, 0)\right\|_{l_{s}^{2}} \\
& \left\|e^{i n^{3} t} q(n, 0)-e^{i n^{3} t} u(n, 0)\right\|_{l_{s}^{2}} \lesssim\langle t\rangle \varepsilon^{1-s-} .
\end{aligned}
$$

The first and the third estimates follow from Proposition 2.1 while the second follows from the estimate on the equation (13).

This inequality for $s=0$ implies that $\|u(\cdot, t)\|_{\ell^{2}} \leq 2 \rho \sqrt{\varepsilon}$ for $t \lesssim \varepsilon^{-1 / 2+}$, while the conservation of Hamiltonian implies that $\|u(\cdot, t)\|_{\ell_{3 / 2}^{2}} \leq 2 \rho \varepsilon^{-1}$. Therefore, $u$ stays in $X_{\varepsilon}^{2 \rho}$ up to the time $t \lesssim \varepsilon^{-1 / 2+}$. This is important since our estimates for the canonical transformations are only valid in the ball $X_{\varepsilon}^{C \rho}$.

Moreover, for $s=1 / 2$, the last inequality gives (8). This ends the proof of Theorem 2.2.

\section{Notation.}

- We always assume by default that the summation index avoids the terms with vanishing denominators, and that the summation indices do not vanish. To illustrate this notation, consider the example

$$
\sum_{n_{1}+n_{2}+n_{3}=0} \frac{f\left(n_{1}, n_{2}, n_{3}\right)}{n_{1}\left(n_{1}-n_{2}\right)}:=\sum_{\substack{n_{1}+n_{2}+n_{3}=0 \\ n_{1} \neq 0, n_{2} \neq 0, n_{3} \neq 0, n_{1} \neq n_{2}}} \frac{f\left(n_{1}, n_{2}, n_{3}\right)}{n_{1}\left(n_{1}-n_{2}\right)} .
$$

- The expressions under the square roots are always taken over the absolute values, i.e. $\sqrt{f}:=\sqrt{|f|}$.

- $\partial_{q} F$ is the sequence $\frac{\partial F}{\partial q(-n)}$.

- We use $\lesssim$ sign to avoid using unimportant constants:

$A \lesssim B$ means there is an absolute constant $K$ such that $A \leq K B$. In some cases the constant will depend on parameters such as $s$. $A \lesssim B(\eta-)$ means that for any $\gamma>0, A \leq C_{\gamma} B(\eta-\gamma)$. $A \lesssim B(\eta+)$ is defined similarly. 
- $\langle n\rangle=\sqrt{1+n^{2}}$

- We denote the $k$ th derivative of $H$ over the flow of $F$ by $g_{F}^{k} H$, which is defined iteratively as follows

$g_{F}^{0} H:=H, \quad g_{F} H=g_{F}^{1} H=\{H, F\}, \quad g_{F}^{k} H:=\left\{g_{F}^{k-1} H, F\right\}, k=2,3,4, \ldots$

\section{Canonical transformations}

The goal of this section is to transform the Hamiltonian to a more convenient (so called normal) form where the most essential (resonant) terms are left at the low order. The non-resonant terms will be absorbed into appropriate canonical transformations. Resonant terms are those that are constant over the linear Hamiltonian flow, generated by $\Lambda_{2}$, see the formal definition below. In this section, the separation into the higher and lower order terms is formal as we will not invoke any estimates, yet. The results of this section are not new and follow closely the standard normal form calculations, see e.g. [6, 8].

Consider the change of variables $u=u(q)$, generated by the time- 1 flow of a purely imaginary Hamiltonian $F$. Namely, solve

$$
\frac{d w(n)}{d \tau}=\sigma(n) \frac{\partial F}{\partial w(-n)}, \quad n \neq 0
$$

with initial conditions

$$
\left.w\right|_{\tau=0}=q,
$$

thus producing a symplectic transformation $u=u(q):=\Phi_{F}^{1}(q)=\left.w\right|_{\tau=1}$. On the other hand, we can write $q=\Phi_{F}^{-1}(u)$. Let $\Phi_{F}^{\tau}$ be the time $\tau$ map of the flow of $F$. Using Taylor expansion in $\tau$, evaluated at $\tau=1$, we have

$$
\begin{aligned}
H \circ \Phi_{F}^{1}(q) & =H(q)+\{H, F\}(q)+\ldots+\frac{1}{k !}\{\ldots\{\{H, \underbrace{F\}, F\}, \ldots, F}_{k}\}(q) \\
& +\int_{0}^{1} \frac{(1-\tau)^{k}}{k !}\left\{\ldots \left\{\{H, \underbrace{F\}, F\}, \ldots, F}_{k+1}\} \circ \Phi_{F}^{\tau}(q) d \tau,\right.\right.
\end{aligned}
$$

where the Poisson bracket is defined as the derivative of one Hamiltonian function, over the flow of the other one

$$
\{A, B\}=\sum_{n \neq 0} \sigma(n) \frac{\partial A}{\partial q(n)} \frac{\partial B}{\partial q(-n)} .
$$

Using the notation $g_{F}^{k}(H)$, see above, we can rewrite (15) as

$$
\begin{aligned}
& H \circ \Phi_{F}^{1}= \\
& H+g_{F} H+\frac{1}{2} g_{F}^{2} H+\ldots+\frac{1}{k !} g_{F}^{k} H+\int_{0}^{1} \frac{(1-\tau)^{k}}{k !}\left(g_{F}^{k+1} H\right) \circ \Phi_{F}^{\tau} d \tau .
\end{aligned}
$$


We choose the first transformation as a time-1 map of the Hamiltonian flow of purely imaginary Hamiltonian function

$$
F_{1}=\sum_{n_{1}+n_{2}+n_{3}=0} \mathcal{F}_{1}\left(n_{1}, n_{2}, n_{3}\right) u\left(n_{1}\right) u\left(n_{2}\right) u\left(n_{3}\right) .
$$

With this choice of symplectic structure, all Hamiltonian functions must be purely imaginary. In particular, this Hamiltonian function is purely imaginary provided

$$
\mathcal{F}_{1}\left(-n_{1},-n_{2},-n_{3}\right)=\overline{\mathcal{F}_{1}\left(n_{1}, n_{2}, n_{3}\right)} .
$$

Using (17) with $k=2$ we have

$$
H \circ \Phi_{F_{1}}^{1}=H+g_{F_{1}} H+\frac{1}{2} g_{F_{1}}^{2} H+\frac{1}{2} \int_{0}^{1}(1-\tau)^{2}\left(g_{F_{1}}^{3} H\right) \circ \Phi_{F_{1}}^{\tau} d \tau .
$$

Definition 3.1. The monomial $M_{n_{1} n_{2} \ldots n_{k}}=q\left(n_{1}\right) q\left(n_{2}\right) \ldots q\left(n_{k}\right)$ is called resonant if it commutes with the linear flow, i.e.

$$
\left\{\Lambda_{2}, M\right\}=0 \text {. }
$$

Otherwise, the monomial is called non-resonant. The sum of monomials is called resonant (non-resonant) if all monomials are resonant (nonresonant). We will write \{\}$=\{\}^{\mathrm{r}}+\{\}^{\mathrm{nr}}$, where \{\}$^{\mathrm{r}}$ represents resonant terms and \{\}$^{\mathrm{nr}}$ represents nonresonant terms.

Rewriting the Hamiltonian with $H=\Lambda_{2}+H_{3}$, we have

$$
\begin{aligned}
& H \circ \Phi_{F_{1}}^{1}= \\
& \Lambda_{2}+H_{3}+\left\{\Lambda_{2}, F_{1}\right\}+\left\{H_{3}, F_{1}\right\}+\frac{1}{2}\left\{\left\{\Lambda_{2}, F_{1}\right\}, F_{1}\right\}+\frac{1}{2}\left\{\left\{H_{3}, F_{1}\right\}, F_{1}\right\}+R_{1},
\end{aligned}
$$

where

$$
R_{1}=\frac{1}{2} \int_{0}^{1}(1-\tau)^{2}\left(g_{F_{1}}^{3} H\right) \circ \Phi_{F_{1}}^{\tau} d \tau .
$$

We choose $F_{1}$ so that to eliminate cubic non-resonant terms (in our case all cubic terms are non-resonant)

$$
\left\{\Lambda_{2}, F_{1}\right\}+H_{3}=0 .
$$

Then we have

$$
H \circ \Phi_{F_{1}}^{1}=\Lambda_{2}+\frac{1}{2}\left\{H_{3}, F_{1}\right\}+\frac{1}{2}\left\{\left\{H_{3}, F_{1}\right\}, F_{1}\right\}+R_{1} .
$$

It turns out that another transformation $F_{2}$ that removes non-resonant terms in $\left\{H_{3}, F_{1}\right\}$ is required. For this purpose, we choose $F_{2}$ so that

$$
\left\{\Lambda_{2}, F_{2}\right\}+\frac{1}{2}\left\{H_{3}, F_{1}\right\}=\frac{1}{2}\left\{H_{3}, F_{1}\right\}^{\mathrm{r}}=\frac{3}{2} i \sum_{n \neq 0}|q(n)|^{4},
$$


see (30). Applying (15) with $k=1$, the new Hamiltonian takes the form

$$
\begin{aligned}
H \circ \Phi_{F_{1}}^{1} \circ \Phi_{F_{2}}^{1}= & \Lambda_{2}+\frac{1}{2}\left\{H_{3}, F_{1}\right\}+\frac{1}{2}\left\{\left\{H_{3}, F_{1}\right\}, F_{1}\right\}+R_{1} \\
& +\left\{\Lambda_{2}, F_{2}\right\}+\frac{1}{2}\left\{\left\{H_{3}, F_{1}\right\}, F_{2}\right\}+\frac{1}{2}\left\{\left\{\left\{H_{3}, F_{1}\right\}, F_{1}\right\}, F_{2}\right\} \\
& +\left\{R_{1}, F_{2}\right\}+\int_{0}^{1}(1-\tau)\left\{\left\{H \circ \Phi_{F_{1}}^{1}, F_{2}\right\}, F_{2}\right\} \circ \Phi_{F_{2}}^{\tau} d \tau .
\end{aligned}
$$

Using (23), we rewrite

$$
H \circ \Phi_{F_{1}}^{1} \circ \Phi_{F_{2}}^{1}=\Lambda_{2}+R,
$$

where

$$
\begin{aligned}
R= & i \frac{3}{2} \sum_{n \neq 0}|q(n)|^{4}+\frac{1}{2} g_{F_{1}}^{2} H_{3}+\frac{1}{2} g_{F_{2}} g_{F_{1}} H_{3}+\frac{1}{2} g_{F_{2}}^{2} g_{F_{1}} H_{3} \\
& +R_{1}+g_{F_{2}} R_{1}+\int_{0}^{1}(1-\tau) g_{F_{2}}^{2}\left(H \circ \Phi_{F_{1}}^{1}\right) \circ \Phi_{F_{2}}^{\tau} d \tau,
\end{aligned}
$$

and $R_{1}$ is given by (20).

3.1. Calculation of $F_{1}$. Straightforward calculations give

$$
\left\{\Lambda_{2}, F_{1}\right\}=i \sum_{n_{1}+n_{2}+n_{3}=0}\left(n_{1}^{3}+n_{2}^{3}+n_{3}^{3}\right) \mathcal{F}_{1}\left(n_{1}, n_{2}, n_{3}\right) q\left(n_{1}\right) q\left(n_{2}\right) q\left(n_{3}\right)
$$

Note that under the restriction $n_{1}+n_{2}+n_{3}=0$, the sum of cubes can be factored out

$$
n_{1}^{3}+n_{2}^{3}+n_{3}^{3}=3 n_{1} n_{2} n_{3} .
$$

Thus, from (21) we have

$$
\mathcal{F}_{1}\left(n_{1}, n_{2}, n_{3}\right)=-\frac{\sigma\left(n_{1} n_{2} n_{3}\right)}{3 \sqrt{n_{1} n_{2} n_{3}}},
$$

whenever $n_{1} n_{2} n_{3} \neq 0$. Otherwise $\mathcal{F}\left(n_{1}, n_{2}, n_{3}\right)=0$.

3.2. Calculation of $F_{2}$. We need to solve

$$
\left\{\Lambda_{2}, F_{2}\right\}+\frac{1}{2}\left\{H_{3}, F_{1}\right\}^{\mathrm{nr}}=0,
$$

but first we need to distinguish the resonant and nonresonant terms of $\left\{H_{3}, F_{1}\right\}$ :

$$
\left\{H_{3}, F_{1}\right\}=\left\{H_{3}, F_{1}\right\}^{\mathrm{nr}}+\left\{H_{3}, F_{1}\right\}^{\mathrm{r}}
$$

Recall,

$$
F_{1}=-\sum_{n_{1}+n_{2}+n_{3}=0} \frac{\sigma\left(n_{1} n_{2} n_{3}\right)}{3 \sqrt{n_{1} n_{2} n_{3}}} q\left(n_{1}\right) q\left(n_{2}\right) q\left(n_{3}\right)
$$

and

$$
H_{3}(q)=i \sum_{n_{1}+n_{2}+n_{3}=0} \sqrt{n_{1} n_{2} n_{3}} q\left(n_{1}\right) q\left(n_{2}\right) q\left(n_{3}\right) .
$$


Now, we compute

$$
\begin{aligned}
& \left\{H_{3}, F_{1}\right\}=\sum_{n \neq 0} \sigma(n) \frac{\partial H_{3}}{\partial q(n)} \frac{\partial F_{1}}{\partial q(-n)} \\
& =-i \sum_{n \neq 0} \sigma(n) 3 \sum_{n_{1}+n_{2}+n=0} \sqrt{n_{1} n_{2} n} q\left(n_{1}\right) q\left(n_{2}\right) \sum_{k_{1}+k_{2}-n=0} \frac{\sigma\left(k_{1} k_{2} n\right)}{\sqrt{k_{1} k_{2} n}} q\left(k_{1}\right) q\left(k_{2}\right) \\
& =-3 i \sum_{\substack{n_{1}+n_{2}+n_{3}+n_{4}=0 \\
n_{1}+n_{2} \neq 0}} \sqrt{\frac{n_{1} n_{2}}{n_{3} n_{4}}} \sigma\left(n_{3} n_{4}\right) q\left(n_{1}\right) q\left(n_{2}\right) q\left(n_{3}\right) q\left(n_{4}\right) .
\end{aligned}
$$

The resonant terms are the ones satisfying $n_{1}^{3}+n_{2}^{3}+n_{3}^{3}+n_{4}^{3}=0$. Since we can rewrite (under the restriction $n_{1}+n_{2}+n_{3}+n_{4}=0$ )

$$
n_{1}^{3}+n_{2}^{3}+n_{3}^{3}+n_{4}^{3}=3\left(n_{1}+n_{2}\right)\left(n_{1}+n_{3}\right)\left(n_{2}+n_{3}\right)
$$

and $n_{1}+n_{2} \neq 0$, the resonant terms are the ones with $n_{1}+n_{3}=0$ or $n_{2}+n_{3}=0$. Therefore, the nonresonant terms are

$$
\left\{H_{3}, F_{1}\right\}^{\mathrm{nr}}=-3 i \sum_{\substack{n_{1}+n_{3} \neq 0, n_{2}+n_{3} \neq 0, n_{1}+n_{2} \neq 0 \\ n_{1}+n_{2}+n_{3}+n_{4}=0}} \sqrt{\frac{n_{1} n_{2}}{n_{3} n_{4}}} \sigma\left(n_{3} n_{4}\right) q\left(n_{1}\right) q\left(n_{2}\right) q\left(n_{3}\right) q\left(n_{4}\right) .
$$

On the other hand, the resonant terms are

$$
\begin{aligned}
\left\{H_{3}, F_{1}\right\}^{\mathrm{r}} & =-3 i \sum_{\substack{n_{1}+n_{3}=0, n_{1}+n_{2} \neq 0 \\
n_{1}+n_{2}+n_{3}+n_{4}=0}}(\cdots)-3 i \sum_{\substack{n_{2}+n_{3}=0, n_{1}+n_{3} \neq 0, n_{1}+n_{2} \neq 0 \\
n_{1}+n_{2}+n_{3}+n_{4}=0}}(\cdots) \\
& =-3 i \sum_{n_{1}+n_{2} \neq 0} \sigma\left(n_{1} n_{2}\right)\left|q\left(n_{1}\right)\right|^{2}\left|q\left(n_{2}\right)\right|^{2}-3 i \sum_{n_{1} \pm n_{2} \neq 0} \sigma\left(n_{1} n_{2}\right)\left|q\left(n_{1}\right)\right|^{2}\left|q\left(n_{2}\right)\right|^{2} .
\end{aligned}
$$

The resonant terms can be simplified as follows

$$
(29)=-6 i \sum_{n_{1} \pm n_{2} \neq 0} \sigma\left(n_{1} n_{2}\right)\left|q\left(n_{1}\right)\right|^{2}\left|q\left(n_{2}\right)\right|^{2}+3 i \sum_{n \neq 0}|q(n)|^{4}=3 i \sum_{n \neq 0}|q(n)|^{4} \text {, }
$$

since the first sum is equal to zero due to the cancellations

$$
\sigma\left(n_{1} n_{2}\right)+\sigma\left(-n_{1} n_{2}\right)=0
$$

and $|q(m)|=|q(-m)|$.

Therefore, we have

$$
\left\{H_{3}, F_{1}\right\}^{\mathrm{r}}=3 i \sum_{n \neq 0}|q(n)|^{4}
$$

Next, we solve

$$
\left\{\Lambda_{2}, F_{2}\right\}+\frac{1}{2}\left\{H_{3}, F_{1}\right\}^{\mathrm{nr}}=0 .
$$

By straightforward calculations, taking $F_{2}$ of the form

$$
F_{2}=\sum_{n_{1}+n_{2}+n_{3}+n_{4}=0} \mathcal{F}_{2}\left(n_{1}, n_{2}, n_{3}, n_{4}\right) q\left(n_{1}\right) q\left(n_{2}\right) q\left(n_{3}\right) q\left(n_{4}\right),
$$


we obtain

$$
\begin{aligned}
& \left\{\Lambda_{2}, F_{2}\right\}= \\
& =i \sum_{n_{1}+n_{2}+n_{3}+n_{4}=0}\left(n_{1}^{3}+n_{2}^{3}+n_{3}^{3}+n_{4}^{3}\right) \mathcal{F}_{2}\left(n_{1}, n_{2}, n_{3}, n_{4}\right) q\left(n_{1}\right) q\left(n_{2}\right) q\left(n_{3}\right) q\left(n_{4}\right) .
\end{aligned}
$$

Therefore, $\mathcal{F}_{2}$ must satisfy the equality

$$
\left(n_{1}^{3}+n_{2}^{3}+n_{3}^{3}+n_{4}^{3}\right) \mathcal{F}_{2}\left(n_{1}, n_{2}, n_{3}, n_{4}\right)-\frac{3}{2} \sqrt{\frac{n_{1} n_{2}}{n_{3} n_{4}}} \sigma\left(n_{3} n_{4}\right)=0,
$$

on the "non-resonant set"

$N R_{4}=\left\{\left(n_{1}, n_{2}, n_{3}, n_{4}\right) \in \mathbb{Z}^{4}, n_{1}+n_{2}+n_{3}+n_{4}=0, n_{1}^{3}+n_{2}^{3}+n_{3}^{3}+n_{4}^{3} \neq 0\right\}$.

Thus,

$$
\mathcal{F}_{2}\left(n_{1}, n_{2}, n_{3}, n_{4}\right)=\frac{3}{2} \sqrt{\frac{n_{1} n_{2}}{n_{3} n_{4}}} \sigma\left(n_{3} n_{4}\right) \frac{1}{n_{1}^{3}+n_{2}^{3}+n_{3}^{3}+n_{4}^{3}},
$$

if $\left(n_{1}, n_{2}, n_{3}, n_{4}\right) \in N R_{4}$, and $\mathcal{F}_{2}=0$ otherwise.

\section{Proof of Proposition 2.1}

We start with a-priori estimates for the derivatives of $F_{1}$ and $F_{2}$. We need these estimates also in the subsequent sections. Define the sequence

$$
f_{1}\left(q_{1}, q_{2}\right)(n):=-\sum_{n_{1}+n_{2}+n=0} \frac{\sigma\left(n_{1} n_{2} n\right)}{\sqrt{n_{1} n_{2} n}} q_{1}\left(n_{1}\right) q_{2}\left(n_{2}\right)
$$

so that

$$
\partial_{q} F_{1}(n)=\frac{\partial F_{1}}{\partial q(-n)}=f_{1}(q, q)(n) .
$$

Lemma 4.1. The following a-priori estimates hold,

$$
\begin{aligned}
\left\|f_{1}\left(q_{1}, q_{2}\right)\right\|_{\ell_{0-}^{2}} & \lesssim\left\|q_{1}\right\|_{\ell_{-1 / 2}^{2}}\left\|q_{2}\right\|_{\ell_{-1 / 2}^{2}}, \\
\left\|f_{1}\left(q_{1}, q_{2}\right)\right\|_{\ell_{\frac{1}{2}}^{2}} & \lesssim\left\|q_{1}\right\|_{\ell_{-1 / 2}^{2}}\left\|q_{2}\right\|_{\ell^{2}}, \\
\left\|f_{1}\left(q_{1}, q_{2}\right)\right\|_{\ell_{1-}^{2}} & \lesssim\left\|q_{1}\right\|_{\ell^{2}}\left\|q_{2}\right\|_{\ell^{2}}, \\
\left\|f_{1}\left(q_{1}, q_{2}\right)\right\|_{\ell_{\frac{3}{2}}^{2}} & \lesssim\left\|q_{1}\right\|_{\ell_{1 / 2}^{2}}\left\|q_{2}\right\|_{\ell_{0+}^{2}}+\left\|q_{1}\right\|_{\ell_{0+}^{2}}\left\|q_{2}\right\|_{\ell_{1 / 2}^{2}} .
\end{aligned}
$$

Now, define the sequence

$$
\begin{aligned}
& f_{2}\left(q_{1}, q_{2}, q_{3}\right)(n)=\partial_{q} F_{2}(n)=\frac{\partial F_{2}}{\partial q(-n)}= \\
& 3 \sum_{n+n_{1}+n_{2}+n_{3}=0} \frac{\sqrt{\frac{n n_{1}}{n_{2} n_{3}}} \sigma\left(n_{2} n_{3}\right)+\sqrt{\frac{n_{1} n_{2}}{n_{3} n}} \sigma\left(n_{3} n\right)}{n^{3}+n_{1}^{3}+n_{2}^{3}+n_{3}^{3}} q_{1}\left(n_{1}\right) q_{2}\left(n_{2}\right) q_{3}\left(n_{3}\right) .
\end{aligned}
$$


Lemma 4.2. For any permutation $\left(j_{1}, j_{2}, j_{3}\right)$ of $(1,2,3)$, and for any $s \in[-1,1]$, we have

$$
\left\|f_{2}\left(q_{1}, q_{2}, q_{3}\right)\right\|_{\ell_{s}^{2}} \lesssim\left\|q_{j_{1}}\right\|_{\ell_{s}^{2}}\left\|q_{j_{2}}\right\|_{\ell_{0+}^{2}}\left\|q_{j_{3}}\right\|_{\ell_{0+}^{2}} .
$$

Moreover,

$$
\left\|f_{2}\left(q_{1}, q_{2}, q_{3}\right)\right\|_{\ell_{3 / 2}^{2}} \lesssim \sum\left\|q_{j_{1}}\right\|_{\ell_{3 / 2}^{2}}\left\|q_{j_{2}}\right\|_{\ell_{0+}^{2}}\left\|q_{j_{3}}\right\|_{\ell_{0+}^{2}}
$$

where the sum is taken over all permutations $\left(j_{1}, j_{2}, j_{3}\right)$ of $(1,2,3)$.

Now, we prove Proposition 2.1 using Lemma 4.1 and Lemma 4.2. The proof of these lemmas will be given in the next section.

Proof of Proposition 2.1. It suffices to prove that $\Phi_{F}^{1}$ is near identity for each $F=F_{1}$ and $F=F_{2}$ in the sense

$$
q \in X_{\varepsilon}^{\rho} \Longrightarrow\left\|\Phi_{F}^{1}(q)-q\right\|_{\ell_{s}^{2}} \lesssim \varepsilon^{1-s-}, s \in[0,3 / 2] .
$$

This is because $\left\|\Phi_{F}^{1}(q)-q\right\|_{\ell_{s}^{2}} \lesssim \varepsilon^{1-s-}$ implies that $\Phi_{F}^{1}(q) \in X_{\varepsilon}^{2 \rho}$ and because if $\Phi_{F_{1}}^{1}$ and $\Phi_{F_{2}}^{1}$ are near identity, then their composition, $\Phi_{F_{1}}^{1} \circ \Phi_{F_{2}}^{1}$, is also near identity.

Note that in light of equation (14) we have

$$
\left\|\Phi_{F_{1}}^{1}(q)-q\right\|_{\ell_{s}^{2}}=\left\|\int_{0}^{1} \sigma(n) \frac{\partial F_{1}}{\partial w(-n)} d \tau\right\|_{\ell_{s}^{2}} \leq\left\|\frac{\partial F_{1}}{\partial w(-n)}\right\|_{\ell_{s}^{2}}=\left\|f_{1}(w, w)\right\|_{\ell_{s}^{2}} .
$$

Applying Lemma 4.1 with $q_{1}=q_{2}=w=\Phi_{F_{1}}^{\tau}(q) \in X_{\varepsilon}^{2 \rho}$, we obtain

$$
\begin{aligned}
\left\|f_{1}(w, w)\right\|_{l^{2}} & \lesssim \varepsilon \\
\left\|f_{1}(w, w)\right\|_{l_{\frac{3}{2}}^{2}} & \lesssim \varepsilon^{\frac{1}{2}-},
\end{aligned}
$$

which implies that $\Phi_{F_{1}}^{1}$ is near identity.

Similarly, applying Lemma 4.2, with $q_{1}=q_{2}=q_{3}=w \in X_{\varepsilon}^{\rho}$ we have

$$
\begin{aligned}
\left\|f_{2}(w, w, w)\right\|_{l^{2}} & \lesssim \varepsilon^{\frac{3}{2}-} \\
\left\|f_{2}(w, w, w)\right\|_{l_{\frac{3}{2}}^{2}} & \lesssim \varepsilon^{0-},
\end{aligned}
$$

which implies that $\Phi_{F_{2}}^{1}$ is near identity.

Since (14) is time reversible, $\Phi_{F_{1}}^{-1}$ and $\Phi_{F_{2}}^{-1}$ are also near identity, which implies that $q(u) \in X_{\varepsilon}^{2 \rho}$ if $u \in X_{\varepsilon}^{\rho}$.

4.1. Proof of Lemma 4.1 and Lemma 4.2. We use the following lemma repeatedly in the proof of Lemma 4.1, Lemma 4.2, and in the subsequent sections. The proof is left to the reader.

Lemma 4.3. a) For any $s, r \in \mathbb{R}, 1 \leq p, q \leq \infty$ and $\frac{1}{p}-\frac{1}{q}>s-r \geq 0$, we have the embedding,

$$
\|u\|_{l_{r}^{p}} \leq C\|u\|_{l_{s}^{q} .}
$$


b) Let $1 \leq p, q, r \leq \infty$ and $\frac{1}{p}+\frac{1}{q}=1+\frac{1}{r}$, we have Young's inequality,

$$
\|u * v\|_{l^{r}} \leq\|u\|_{l^{p}}\|v\|_{l^{q}}
$$

For the convenience of the reader we record the definition of the discrete convolution

$$
u * v(n)=\sum_{m} u(m) v(n-m)=\sum_{m} u(n-m) v(m) .
$$

By a slight abuse of notation we also denote by $u * v$ all the sums of the form $\sum_{m} u(m) v( \pm n-m)$. Young's inequality holds true for all these convolution products of functions.

Proof of Lemma 4.1. We begin with the second estimate. Note that

$$
\left|f_{1}\right| \lesssim \frac{1}{\sqrt{n}}\left(\frac{\left|q_{1}\right|}{\sqrt{\cdot}} * \frac{\left|q_{2}\right|}{\sqrt{\cdot}}\right)(n) .
$$

Therefore, for $s<1 / 2$,

$$
\begin{aligned}
\left\|f_{1}\right\|_{\ell_{s}^{2}} & \lesssim\left\|\frac{\left|q_{1}\right|}{\sqrt{\cdot}} * \frac{\left|q_{2}\right|}{\sqrt{\cdot}}\right\|_{\ell_{s-1 / 2}^{2}} \lesssim\left\|\frac{\left|q_{1}\right|}{\sqrt{\cdot}} * \frac{\left|q_{2}\right|}{\sqrt{\cdot}}\right\|_{\ell^{2+}} \\
& \lesssim\left\|\frac{\left|q_{1}\right|}{\sqrt{\cdot}}\right\|_{\ell^{2}}\left\|\frac{\left|q_{2}\right|}{\sqrt{\cdot}}\right\|_{\ell^{1+}} \lesssim\left\|q_{1}\right\|_{\ell_{-1 / 2}^{2}}\left\|q_{2}\right\|_{\ell^{2}} .
\end{aligned}
$$

The second inequality follows from the first part of Lemma 4.3, and the third inequality follows from Young's inequality. Finally the last inequality is another application of the first part of the aforementioned Lemma.

To prove the first estimate, note that for $s<0$,

$$
\begin{aligned}
\left\|f_{1}\right\|_{\ell_{s}^{2}} & \lesssim\left\|\frac{\left|q_{1}\right|}{\sqrt{\cdot}} * \frac{\left|q_{2}\right|}{\sqrt{\cdot}}\right\|_{\ell_{s-1 / 2}^{2}} \lesssim\left\|\frac{\left|q_{1}\right|}{\sqrt{\cdot}} * \frac{\left|q_{2}\right|}{\sqrt{\cdot}}\right\|_{\ell^{\infty}} \\
& \lesssim\left\|\frac{\left|q_{1}\right|}{\sqrt{\cdot}}\right\|_{\ell^{2}}\left\|\frac{\left|q_{2}\right|}{\sqrt{\cdot}}\right\|_{\ell^{2}}=\left\|q_{1}\right\|_{\ell_{-1 / 2}^{2}}\left\|q_{2}\right\|_{\ell_{-1 / 2}^{2}} .
\end{aligned}
$$

Again the second inequality follows from the first part of Lemma 4.3 and the third inequality follows from Young's inequality.

For the fourth estimate note that for $s>1 / 2$, using $|n|^{s-1 / 2} \lesssim\left|n_{1}\right|^{s-1 / 2}+$ $\left|n_{2}\right|^{s-1 / 2}$, we have

$$
|n|^{s}\left|f_{1}(n)\right| \lesssim\left(|\cdot|^{s-1}\left|q_{1}\right|\right) *\left(|\cdot|^{-1 / 2}\left|q_{2}\right|\right)(n)+\left(|\cdot|^{-1 / 2}\left|q_{1}\right|\right) *\left(|\cdot|^{s-1}\left|q_{2}\right|\right)(n) .
$$

Therefore, for $s>1 / 2$,

$$
\left\|f_{1}\right\|_{\ell_{s}^{2}} \lesssim\left\|\left(|\cdot|^{s-1}\left|q_{1}\right|\right) *\left(|\cdot|^{-1 / 2}\left|q_{2}\right|\right)\right\|_{\ell^{2}}+\left\|\left(|\cdot|^{-1 / 2}\left|q_{1}\right|\right) *\left(|\cdot|^{s-1}\left|q_{2}\right|\right)\right\|_{\ell^{2}} .
$$


In particular, for $s=3 / 2$, we have

$$
\begin{aligned}
\left\|f_{1}\right\|_{\ell_{3 / 2}^{2}} & \lesssim\left\|\left(|\cdot|^{1 / 2}\left|q_{1}\right|\right) *\left(|\cdot|^{-1 / 2}\left|q_{2}\right|\right)\right\|_{\ell^{2}}+\left\|\left(|\cdot|^{-1 / 2}\left|q_{1}\right|\right) *\left(|\cdot|{ }^{1 / 2}\left|q_{2}\right|\right)\right\|_{\ell^{2}} \\
& \lesssim\left\||\cdot|^{1 / 2} q_{1}\right\|_{\ell^{2}}\left\||\cdot|^{-1 / 2} q_{2}\right\|_{\ell^{1}}+\left\||\cdot|^{1 / 2} q_{2}\right\|_{\ell^{2}}\left\||\cdot|^{-1 / 2} q_{1}\right\|_{\ell^{1}} \\
& \lesssim\left\|q_{1}\right\|_{\ell_{1 / 2}^{2}}\left\|q_{2}\right\|_{\ell_{0+}^{2}}+\left\|q_{2}\right\|_{\ell_{1 / 2}^{2}}\left\|q_{1}\right\|_{\ell_{0+}^{2}}
\end{aligned}
$$

For this estimate we first apply Young's inequality and then the first part of Lemma 4.3 .

Finally to prove the third estimate, for fixed $\delta>0$

$$
\begin{aligned}
\left\|f_{1}\right\|_{\ell_{1-\delta}^{2}} & \lesssim\left\|\left(|\cdot|^{-\delta}\left|q_{1}\right|\right) *\left(|\cdot|^{-1 / 2}\left|q_{2}\right|\right)\right\|_{\ell^{2}}+\left\|\left(|\cdot|^{-1 / 2}\left|q_{1}\right|\right) *\left(|\cdot|^{-\delta}\left|q_{2}\right|\right)\right\|_{\ell^{2}} \\
& \lesssim\left\||\cdot|^{-\delta} q_{1}\right\|_{\ell^{2-\eta}}\left\||\cdot|^{-1 / 2} q_{2}\right\|_{\ell^{1+\tilde{\eta}}}+\left\||\cdot|^{-\delta} q_{2}\right\|_{\ell^{2-\eta}}\left\||\cdot|^{-1 / 2} q_{1}\right\|_{\ell^{1+\tilde{\eta}}} \\
& \lesssim\left\|q_{1}\right\|_{\ell^{2}}\left\|q_{2}\right\|_{\ell^{2} .}
\end{aligned}
$$

The derivation of this last string of inequalities follows as above using Lemma 4.3. We only note that the last step follows if we choose $\eta, \tilde{\eta}>0$ sufficiently small with $\eta$ depending on $\delta>0$.

Proof of Lemma 4.2. First note that by duality and interpolation it suffices to prove the first statement for $s=1$. To estimate $\left\|f_{2}\right\|_{\ell_{1}^{2}}$ we use duality as follows

$$
\left\|f_{2}\right\|_{\ell_{1}^{2}}=\sup _{\|h\|_{\ell_{-1}^{2}=1}}\left|\left\langle f_{2}, h\right\rangle\right|
$$

Note that for any permutation $\left(j_{1}, j_{2}, j_{3}\right)$ the form $\left\langle f_{2}, h\right\rangle$ on the right hand side can be estimated by

$$
\begin{aligned}
\sum_{n_{1}+n_{2}+n_{3}+n_{4}=0} \frac{\left|n_{1} n_{2}\right|+\left|n_{1} n_{3}\right|+\left|n_{1} n_{4}\right|+}{\sqrt{n_{1} n_{2} n_{3} n_{4}}\left|n_{1}^{3}+n_{2}^{3}+n_{3}^{3}+n_{4}^{3}\right|} & \\
\times & \left|q_{j_{1}}\left(n_{1}\right) q_{j_{2}}\left(n_{2}\right) q_{j_{3}}\left(n_{3}\right) h\left(n_{4}\right)\right| .
\end{aligned}
$$

Since $q_{j_{2}}$ and $q_{j_{3}}$ appear symmetrically on the right side of the inequality, it suffices to estimate the following sum

$$
\sum_{n_{1}+n_{2}+n_{3}+n_{4}=0} \frac{\left|n_{1} n_{2}\right|+\left|n_{1} n_{4}\right|+\left|n_{2} n_{3}\right|+\left|n_{2} n_{4}\right|}{\sqrt{n_{1} n_{2} n_{3} n_{4}}\left|n_{1}^{3}+n_{2}^{3}+n_{3}^{3}+n_{4}^{3}\right|}\left|q_{j_{1}}\left(n_{1}\right) q_{j_{2}}\left(n_{2}\right) q_{j_{3}}\left(n_{3}\right) h\left(n_{4}\right)\right| .
$$

The estimate for these summands are very similar to each other, therefore we consider only two of them. The remaining estimates just recycle the arguments below and will be omitted:

$$
\sum_{n_{1}+n_{2}+n_{3}+n_{4}=0} \frac{\left|n_{2} n_{3}\right|+\left|n_{2} n_{4}\right|}{\sqrt{n_{1} n_{2} n_{3} n_{4}}\left|n_{1}^{3}+n_{2}^{3}+n_{3}^{3}+n_{4}^{3}\right|}\left|q_{j_{1}}\left(n_{1}\right) q_{j_{2}}\left(n_{2}\right) q_{j_{3}}\left(n_{3}\right) h\left(n_{4}\right)\right|
$$




$$
\begin{aligned}
&(32)=\sum_{n_{1}+n_{2}+n_{3}+n_{4}=0} \frac{\left|n_{2}\right|^{1 / 2-\delta}\left|n_{3}\right|^{1 / 2-\delta}\left|n_{4}\right|^{1 / 2}}{\left|n_{1}\right|^{3 / 2}\left|n_{1}^{3}+n_{2}^{3}+n_{3}^{3}+n_{4}^{3}\right|} \times \\
& \times\left.\left|n_{1} q_{j_{1}}\left(n_{1}\right)\right| n_{2}\right|^{\delta} q_{j_{2}}\left(n_{2}\right)\left|n_{3}\right|^{\delta} q_{j_{3}}\left(n_{3}\right) \frac{h\left(n_{4}\right)}{n_{4}} \mid \\
&(33)+\sum_{n_{1}+n_{2}+n_{3}+n_{4}=0} \frac{\left|n_{2}\right|^{1 / 2-\delta}\left|n_{4}\right|^{3 / 2}}{\left|n_{1}\right|^{3 / 2}\left|n_{3}\right|{ }^{1 / 2+\delta}\left|n_{1}^{3}+n_{2}^{3}+n_{3}^{3}+n_{4}^{3}\right|} \times \\
& \times\left.\left|n_{1} q_{j_{1}}\left(n_{1}\right)\right| n_{2}\right|^{\delta} q_{j_{2}}\left(n_{2}\right)\left|n_{3}\right|^{\delta} q_{j_{3}}\left(n_{3}\right) \frac{h\left(n_{4}\right)}{n_{4}} \mid .
\end{aligned}
$$

After substituting $n_{4}=-n_{1}-n_{2}-n_{3}$, the multiplier in (33) takes the form

$$
\begin{aligned}
& \frac{\left|n_{2}\right|^{1 / 2-\delta}\left|n_{1}+n_{2}+n_{3}\right|^{3 / 2}}{\left|n_{1}\right|^{3 / 2}\left|n_{3}\right|^{1 / 2+\delta}\left|n_{1}+n_{2}\right|\left|n_{1}+n_{3}\right|\left|n_{2}+n_{3}\right|} \\
& \lesssim \frac{\left|n_{2}\right|^{1 / 2-\delta}}{\left|n_{3}\right|^{1 / 2+\delta}\left|n_{1}+n_{2}\right|\left|n_{1}+n_{3}\right|\left|n_{2}+n_{3}\right|}+\frac{\left|n_{2}\right|^{1 / 2-\delta}\left|n_{2}+n_{3}\right|^{1 / 2}}{\left|n_{1}\right|^{3 / 2}\left|n_{3}\right|^{1 / 2+\delta}\left|n_{1}+n_{2}\right|\left|n_{1}+n_{3}\right|} .
\end{aligned}
$$

Note that $\left|n_{2}\right| \lesssim\left|n_{1}+n_{2}\right|\left|n_{1}+n_{3}\right|\left|n_{2}+n_{3}\right|$ and $\left|n_{2}\right| \lesssim\left|n_{1}\right|\left|n_{1}+n_{2}\right|$ under the condition $\left|n_{1} n_{2} n_{3}\right|\left|n_{1}+n_{2}\right|\left|n_{1}+n_{3}\right|\left|n_{2}+n_{3}\right| \neq 0$. Using this we further bound the multiplier by

$$
\begin{aligned}
& \frac{1}{\left|n_{2} n_{3}\right|^{1 / 2+\delta}}+\frac{\left|n_{2}+n_{3}\right|^{1 / 2}}{\sqrt{n_{1}}\left|n_{2} n_{3}\right|^{1 / 2+\delta}\left|n_{1}+n_{3}\right|} \\
& \lesssim \frac{1}{\left|n_{2} n_{3}\right|^{1 / 2+\delta}}+\frac{1}{\sqrt{n_{1}}\left|n_{3}\right|^{1 / 2+\delta}\left|n_{1}+n_{3}\right|}+\frac{1}{\sqrt{n_{1}}\left|n_{2}\right|^{1 / 2+\delta}\left|n_{3}\right|^{\delta}\left|n_{1}+n_{3}\right|} \\
& \quad \lesssim \frac{1}{\left|n_{2} n_{3}\right|^{1 / 2+\delta}}+\frac{1}{\left|n_{1} n_{3}\right|^{1 / 2+\delta / 2}}+\frac{1}{\left|n_{1} n_{2}\right|^{1 / 2+\delta}} .
\end{aligned}
$$

The last inequality follows from $\left|n_{1}\right| \lesssim\left|n_{3}\right|\left|n_{1}+n_{3}\right|$. The contribution of the first summand in (34) to (33) is

$$
\begin{aligned}
&\left.\sum_{n_{1}+n_{2}+n_{3}+n_{4}=0} \frac{1}{\left|n_{2} n_{3}\right|^{1 / 2+\delta}}\left|n_{1} q_{j_{1}}\left(n_{1}\right)\right| n_{2}\right|^{\delta} q_{j_{2}}\left(n_{2}\right)\left|n_{3}\right|^{\delta} q_{j_{3}}\left(n_{3}\right) \frac{h\left(n_{4}\right)}{n_{4}} \mid \\
&=\left.\sum_{n_{1}, n_{2}, n_{3}} \frac{1}{\left.\left|n_{2} n_{3}\right|\right|^{1 / 2+\delta}}\left|n_{1} q_{j_{1}}\left(n_{1}\right)\right| n_{2}\right|^{\delta} q_{j_{2}}\left(n_{2}\right)\left|n_{3}\right|^{\delta} q_{j_{3}}\left(n_{3}\right) \frac{h\left(-n_{1}-n_{2}-n_{3}\right)}{n_{1}+n_{2}+n_{3}} \mid \\
& \lesssim\left.\left\|q_{j_{1}}\right\|_{\ell_{1}^{2}}\|h\|_{\ell_{-1}^{2}} \sum_{n_{2}, n_{3}} \frac{1}{\left|n_{2} n_{3}\right|^{1 / 2+\delta}}|| n_{2}\right|^{\delta} q_{j_{2}}\left(n_{2}\right)\left|n_{3}\right|^{\delta} q_{j_{3}}\left(n_{3}\right) \mid \\
& \lesssim\left\|q_{j_{1}}\right\|_{\ell_{1}^{2}}\left\|q_{j_{2}}\right\|_{\ell_{\delta}^{2}}\left\|q_{j_{3}}\right\|_{\ell_{\delta}^{2}}\|h\|_{\ell_{-1}^{2}} .
\end{aligned}
$$

The first inequality follows from Cauchy-Schwarz in $n_{1}$ sum and the second follows from Cauchy-Schwarz in $n_{2}, n_{3}$ sums since $\frac{1}{\left|n_{2} n_{3}\right|^{1 / 2+\delta}}$ is square summable. The contribution of the other two summands in (34) to (33) can be estimated similarly. 
Now we consider (32). After substituting $n_{4}=-n_{1}-n_{2}-n_{3}$, the multiplier takes the form

$$
\begin{aligned}
& \frac{\left|n_{2} n_{3}\right|^{1 / 2-\delta}\left|n_{1}+n_{2}+n_{3}\right|^{1 / 2}}{\left|n_{1}\right|^{3 / 2}\left|n_{1}+n_{2}\right|\left|n_{1}+n_{3}\right|\left|n_{2}+n_{3}\right|} \\
& \lesssim \frac{\left|n_{2} n_{3}\right|^{1 / 2-\delta}}{\left|n_{1}\right|\left|n_{1}+n_{2}\right|\left|n_{1}+n_{3}\right|\left|n_{2}+n_{3}\right|}+\frac{\left|n_{2} n_{3}\right|^{1 / 2-\delta}}{\left|n_{1}\right|^{3 / 2}\left|n_{1}+n_{2}\right|\left|n_{1}+n_{3}\right|\left|n_{2}+n_{3}\right|^{1 / 2}} \\
& \lesssim \frac{1}{\left|n_{1}\right|^{2 \delta}\left|n_{1}+n_{2}\right|^{1 / 2+\delta}\left|n_{1}+n_{3}\right|^{1 / 2+\delta}\left|n_{2}+n_{3}\right|}+\frac{1}{\left|n_{2}\right|^{1 / 2+\delta}\left|n_{3}\right|^{\delta}\left|n_{2}+n_{3}\right|^{1 / 2}} \\
& \lesssim \frac{1}{\left|n_{2}-n_{3}\right|^{1 / 2+\delta}\left|n_{2}+n_{3}\right|}+\frac{1}{\left|n_{2}\right|^{1 / 2+\delta}\left|n_{3}\right|^{\delta}\left|n_{2}+n_{3}\right|^{1 / 2}} .
\end{aligned}
$$

We estimate the two terms separately. By summing first in $n_{1}$ and then using Cauchy-Schwarz inequality in $n_{2}, n_{3}$, to estimate

$$
\begin{aligned}
\sum_{n_{1}, n_{2}, n_{3}} \frac{1}{\left|n_{2}-n_{3}\right|^{1 / 2+\delta}\left|n_{2}+n_{3}\right|} \times \\
\quad \times\left.\left|n_{1} q_{j_{1}}\left(n_{1}\right)\right| n_{2}\right|^{\delta} q_{j_{2}}\left(n_{2}\right)\left|n_{3}\right|^{\delta} q_{j_{3}}\left(n_{3}\right) \frac{h\left(-n_{1}-n_{2}-n_{3}\right)}{n_{1}+n_{2}+n_{3}} \mid
\end{aligned}
$$

it is enough to bound

$$
\sum_{n_{2}, n_{3}} \frac{1}{\left|n_{2}-n_{3}\right|^{1+2 \delta}\left|n_{2}+n_{3}\right|^{2}}
$$

But

$$
\sum_{n_{2}, n_{3}} \frac{1}{\left|n_{2}-n_{3}\right|^{1+2 \delta}\left|n_{2}+n_{3}\right|^{2}}=\sum_{n_{2}, m} \frac{1}{|m|^{1+2 \delta}\left|2 n_{2}+m\right|^{2}}<\infty .
$$

To estimate the second term by the above arguments it is enough to estimate

$$
\sum_{n_{2}, n_{3}} \frac{1}{\left|n_{2}\right|^{1+2 \delta}} \frac{1}{\left|n_{3}\right|^{2 \delta}\left|n_{2}+n_{3}\right|} .
$$

But

$$
\sum_{n_{2}, n_{3}} \frac{1}{\left|n_{2}\right|^{1+2 \delta}} \frac{1}{\left|n_{3}\right|^{2 \delta}\left|n_{2}+n_{3}\right|}=\sum_{n_{2}} \frac{1}{\left|n_{2}\right|^{1+2 \delta}}\left(\sum_{n_{3}} \frac{1}{\left|n_{3}\right|^{2 \delta}\left|n_{2}+n_{3}\right|}\right)
$$

and Hölder's inequality implies that

$$
\left\|n_{3}^{-2 \delta}\left(n_{2}+n_{3}\right)^{-1}\right\|_{l^{1}\left(n_{3}\right)} \lesssim\left\|n_{3}^{-2 \delta}\right\|_{l_{n_{3}}^{\infty-}}\left\|\frac{1}{n_{2}+n_{3}}\right\|_{l^{1+}\left(n_{3}\right)}<\infty
$$

while the rest is summable in $n_{2}$. This finishes the proof of the first assertion of the lemma.

To prove the second assertion we use duality in a similar way. Since 
we have a sum over all possible permutations in the right hand side of the inequality it suffices to consider the following sum

$$
\begin{gathered}
\sum_{n_{1}+n_{2}+n_{3}+n_{4}=0} \frac{\left|n_{1} n_{3}\right|+\left|n_{1} n_{4}\right|}{\sqrt{n_{1} n_{2} n_{3} n_{4}}\left|n_{1}^{3}+n_{2}^{3}+n_{3}^{3}+n_{4}^{3}\right|^{3}}\left|q_{j_{1}}\left(n_{1}\right) q_{j_{2}}\left(n_{2}\right) q_{j_{3}}\left(n_{3}\right) h\left(n_{4}\right)\right| \\
=\sum_{n_{1}+n_{2}+n_{3}+n_{4}=0} \frac{\left|n_{3}\right|^{1 / 2-\delta}\left|n_{4}\right|}{\left|n_{1}\right|\left|n_{2}\right|^{1 / 2+\delta}\left|n_{1}^{3}+n_{2}^{3}+n_{3}^{3}+n_{4}^{3}\right|} \times \\
\times \sum_{n_{1}+n_{2}+n_{3}+n_{4}=0} \frac{\left|n_{1}\right|^{3 / 2} q_{j_{1}}\left(n_{1}\right)\left|n_{2}\right|^{\delta} q_{j_{2}}\left(n_{2}\right)\left|n_{3}\right|^{\delta} q_{j_{3}}\left(n_{3}\right) \frac{h\left(n_{4}\right)}{\left|n_{4}\right|^{3 / 2} \mid}}{\left|n_{1}\right|\left|n_{2} n_{3}\right|^{1 / 2+\delta}\left|n_{1}^{3}+n_{2}^{3}+n_{3}^{3}+n_{4}^{3}\right|} \times \\
\times\left.|| n_{1}\right|^{3 / 2} q_{j_{1}}\left(n_{1}\right)\left|n_{2}\right|^{\delta} q_{j_{2}}\left(n_{2}\right)\left|n_{3}\right|^{\delta} q_{j_{3}}\left(n_{3}\right) \frac{h\left(n_{4}\right)}{\left|n_{4}\right|^{3 / 2} \mid .}
\end{gathered}
$$

As above the proof follows from the following estimate for the multipliers:

$$
\begin{aligned}
\frac{\left|n_{3}\right|^{1 / 2-\delta}\left|n_{4}\right|}{\left|n_{1}\right|\left|n_{2}\right|^{1 / 2+\delta}\left|n_{1}^{3}+n_{2}^{3}+n_{3}^{3}+n_{4}^{3}\right|}+\frac{\left|n_{4}\right|^{2}}{\left|n_{1}\right|\left|n_{2} n_{3}\right|^{1 / 2+\delta}\left|n_{1}^{3}+n_{2}^{3}+n_{3}^{3}+n_{4}^{3}\right|} \\
\lesssim \frac{1}{\left|n_{2} n_{3}\right|^{1 / 2+\delta}} .
\end{aligned}
$$

To prove this inequality we first substitute $n_{4}=-n_{1}-n_{2}-n_{3}$ to obtain

$$
\begin{aligned}
& \frac{\left|n_{3}\right|^{1 / 2-\delta}\left|n_{1}+n_{2}+n_{3}\right|}{\left|n_{1}\right|\left|n_{2}\right|^{1 / 2+\delta}\left|n_{1}+n_{2}\right|\left|n_{1}+n_{3}\right|\left|n_{2}+n_{3}\right|}+ \\
& \quad+\frac{\left|n_{1}+n_{2}+n_{3}\right|^{2}}{\left|n_{1}\right|\left|n_{2} n_{3}\right|^{1 / 2+\delta}\left|n_{1}+n_{2}\right|\left|n_{1}+n_{3}\right|\left|n_{2}+n_{3}\right|} \\
& \lesssim \frac{\left|n_{3}\right|^{1 / 2-\delta}}{\left|n_{2}\right|^{1 / 2+\delta}\left|n_{1}+n_{2}\right|\left|n_{1}+n_{3}\right|\left|n_{2}+n_{3}\right|}+\frac{\left|n_{3}\right|^{1 / 2-\delta}}{\left|n_{1}\right|\left|n_{2}\right|^{1 / 2+\delta}\left|n_{1}+n_{2}\right|\left|n_{1}+n_{3}\right|} \\
& +\frac{\left|n_{1}\right|}{\left|n_{2} n_{3}\right|^{1 / 2+\delta}\left|n_{1}+n_{2}\right|\left|n_{1}+n_{3}\right|\left|n_{2}+n_{3}\right|}+\frac{\left|n_{2}+n_{3}\right|}{\left|n_{1}\right|\left|n_{2} n_{3}\right|^{1 / 2+\delta}\left|n_{1}+n_{2}\right|\left|n_{1}+n_{3}\right|} .
\end{aligned}
$$

Using the inequalities

$$
\begin{aligned}
\left|n_{3}\right|,\left|n_{1}\right| & \lesssim\left|n_{1}+n_{2}\right|\left|n_{1}+n_{3}\right|\left|n_{2}+n_{3}\right| \\
\left|n_{3}\right| & \lesssim\left|n_{1}\right|\left|n_{1}+n_{3}\right| \\
\left|n_{2}+n_{3}\right| & \leq\left|n_{2}\right|+\left|n_{3}\right| \lesssim\left|n_{1}\right|\left|n_{1}+n_{2}\right|+\left|n_{1}\right|\left|n_{1}+n_{3}\right|
\end{aligned}
$$

we see that last line is bounded by $\frac{1}{\left|n_{2} n_{3}\right|^{1 / 2+\delta}}$. This finishes the proof of the lemma by using the methods of the first part. 


\section{Proof of Proposition 2.2}

Note that by (10) and (25), it suffices to prove that if $q \in X_{\varepsilon}^{\rho}$, then, for $s \in[0,1 / 2]$,

$$
\begin{aligned}
&\left\|q(k)^{3}\right\|_{\ell_{s}^{2}} \lesssim \varepsilon^{1-s-} \\
&\left\|\partial_{q} g_{F_{2}}^{b} g_{F_{1}}^{a} H_{3}\right\|_{\ell_{s}^{2}} \lesssim \varepsilon^{1-s-}, \quad \text { if } a \geq 1, a+b \geq 2,
\end{aligned}
$$

and similarly for terms involving integrals.

The inequality (35) is obtained as follows:

$$
\left\|q^{3}\right\|_{\ell_{s}^{2}} \leq\|q\|_{\ell^{\infty}}^{2}\|q\|_{\ell_{s}^{2}} \lesssim \varepsilon \varepsilon^{\frac{1}{2}-s}=\varepsilon^{\frac{3}{2}-s} .
$$

The inequality (36) follows from Theorem 5.1 in the next section, and the estimates for the integral terms are discussed in section 5.2 .

5.1. Estimates for the terms $g_{F_{2}}^{b} g_{F_{1}}^{a} H_{3}$. In this section, we estimate the derivative of the commutators $g_{F_{2}}^{b} g_{F_{1}}^{a} H_{3}$ for $a \geq 1$.

Theorem 5.1. Let $\varepsilon>0$. Assume that $q \in X_{\rho}^{\varepsilon}$. Then for $a \geq 1, b \geq 0$, and $s \in[0,1 / 2]$, we have

$$
\left\|\partial_{q} g_{F_{2}}^{b} g_{F_{1}}^{a} H_{3}\right\|_{\ell_{s}^{2}} \lesssim \varepsilon^{\frac{a}{2}+b-s-} .
$$

With a slight abuse of notation, define

$$
H_{3}\left(q_{1}, q_{2}, q_{3}\right)=i \sum_{n_{1}+n_{2}+n_{3}=0} \sqrt{n_{1} n_{2} n_{3}} q_{1}\left(n_{1}\right) q_{2}\left(n_{2}\right) q_{3}\left(n_{3}\right) .
$$

With this notation, $H_{3}(q)=H_{3}(q, q, q)$. Note that $\left\{H_{3}, F_{1}\right\}$ can be written as

$$
H_{3}\left(\partial_{q} F_{1}, q, q\right)+H_{3}\left(q, \partial_{q} F_{1}, q\right)+H_{3}\left(q, q, \partial_{q} F_{1}\right),
$$

where $\partial_{q} F_{1}$ is the sequence $\frac{\partial F_{1}}{\partial q(-n)}=f_{1}(q, q)(n)$. By symmetry, we can write

$$
\left\{H_{3}, F_{1}\right\}=3 H_{3}\left(f_{1}(q, q), q, q\right)
$$

By the same token, we can write $\left\{\left\{H_{3}, F_{1}\right\}, F_{2}\right\}$ as a sum of the following terms

$$
H_{3}\left(f_{1}(q, q), f_{2}(q, q, q), q\right) \text {, and } H_{3}\left(f_{1}\left(f_{2}(q, q, q), q\right), q, q\right) .
$$

To generalize this to higher order commutators, we define $Q_{a, b}$ as follows. First $Q_{0,0}$ is $q$. To obtain $Q_{a, b}$, start with $Q_{0,0}$ and iteratively, a times, replace one $q$ with $f_{1}(q, q)$, then again iteratively replace one $q$ with $f_{2}(q, q, q)$ $b$ times. Any sequence obtained in this manner is called $Q_{a, b}$. For example $\left\{\left\{H_{3}, F_{1}\right\}, F_{2}\right\}$ can be described as a sum of

$$
H_{3}\left(Q_{1,0}, Q_{0,1}, Q_{0,0}\right) \text {, and } H_{3}\left(Q_{1,1}, Q_{0,0}, Q_{0,0}\right) .
$$


In general, we can write $g_{F_{2}}^{b} g_{F_{1}}^{a} H_{3}$ as a sum of terms of the form

$$
H_{3}\left(Q_{a_{1}, b_{1}}, Q_{a_{2}, b_{2}}, Q_{a_{3}, b_{3}}\right), a_{1}+a_{2}+a_{3}=a, b_{1}+b_{2}+b_{3}=b, a_{j}, b_{j} \in \mathbb{N} .
$$

To estimate $\left\|\partial_{q} g_{F_{2}}^{b} g_{F_{1}}^{a} H_{3}\right\|_{\ell_{s}^{2}}$, we use duality and estimate

$$
\sup _{\|h\|_{\ell_{-s}^{2}}=1}\left|\left\langle\partial_{q} g_{F_{2}}^{b} g_{F_{1}}^{a} H_{3}, h\right\rangle\right| \text {. }
$$

Note that $\left\langle\partial_{q} g_{F_{2}}^{b} g_{F_{1}}^{a} H_{3}, h\right\rangle$ can be written as the commutator $\left\{g_{F_{2}}^{b} g_{F_{1}}^{a} H_{3}, G\right\}$, where $G=G(q)=\sum_{n} h(n) q(n)$. This is because $h=\partial_{q} G$. In light of (37) and (38), we can now write $\left\langle\partial_{q} g_{F_{2}}^{b} g_{F_{1}}^{a} H_{3}, h\right\rangle$ as a sum of terms of the form

$$
H_{3}\left(Q_{a_{1}, b_{1}}^{h}, Q_{a_{2}, b_{2}}, Q_{a_{3}, b_{3}}\right), a_{1}+a_{2}+a_{3}=a, b_{1}+b_{2}+b_{3}=b, a_{j}, b_{j} \in \mathbb{N}
$$

where $Q_{a, b}^{h}$ is obtained from $Q_{a, b}$ by replacing one $q$ by $h$. Therefore, to prove Theorem 5.1, we need to estimate the sequences $Q_{a, b}$ and $Q_{a, b}^{h}$ in $\ell_{s}^{2}$ spaces, and estimate $H_{3}\left(q_{1}, q_{2}, q_{3}\right)$ for $q_{j}$ in $\ell_{s}^{2}$ spaces:

Proposition 5.1. For any permutation $\left(j_{1}, j_{2}, j_{3}\right)$ of $(1,2,3)$, we have

$$
\begin{aligned}
\left|H_{3}\left(q_{1}, q_{2}, q_{3}\right)\right| & \lesssim\left\|q_{j_{1}}\right\|_{\ell^{2}}\left\|q_{j_{2}}\right\|_{\ell_{1+}^{2}}\left\|q_{j_{3}}\right\|_{\ell_{1+}^{2}}, \\
\left|H_{3}\left(q_{1}, q_{2}, q_{3}\right)\right| & \lesssim\left\|q_{j_{1}}\right\|_{\ell_{1-}^{2}}\left\|q_{j_{2}}\right\|_{\ell_{1 / 2+}^{2}}\left\|q_{j_{3}}\right\|_{\ell_{1 / 2+}^{2}}, \\
\left|H_{3}\left(q_{1}, q_{2}, q_{3}\right)\right| & \lesssim\left\|q_{j_{1}}\right\|_{\ell_{-1 / 2}^{2}}\left(\left\|q_{j_{2}}\right\|_{\ell_{3 / 2}^{2}}\left\|q_{j_{3}}\right\|_{\ell_{1+}^{2}}+\left\|q_{j_{2}}\right\|_{\ell_{1+}^{2}}\left\|q_{j_{3}}\right\|_{\ell_{3 / 2}^{2}}\right), \\
\left|H_{3}\left(q_{1}, q_{2}, q_{3}\right)\right| & \lesssim\left\|q_{j_{1}}\right\|_{\ell_{1 / 2-}^{2}}\left\|q_{j_{2}}\right\|_{\ell_{1}^{2}}\left\|q_{j_{3}}\right\|_{\ell_{1 / 2+}^{2}}
\end{aligned}
$$

Proposition 5.2. Assume that q satisfies the hypothesis of Theorem 5.1. Then we have

$$
\begin{aligned}
& \left\|Q_{a, b}\right\|_{\ell_{s}^{2}} \lesssim \varepsilon^{\frac{3}{2}-s+\frac{a}{2}+b-}, \quad s \in[1,3 / 2], a \geq 1, b \geq 0 \\
& \left\|Q_{0, b}\right\|_{\ell_{s}^{2}} \lesssim \varepsilon^{\frac{1}{2}-s+b-}, \quad s \in[0,3 / 2], b \geq 0 .
\end{aligned}
$$

Proposition 5.3. Assume that $q$ satisfies the hypothesis of Theorem 5.1. Then for any $a \geq 1, b \geq 0$, we have

$$
\begin{aligned}
\left\|Q_{a, b}^{h}\right\|_{\ell_{1-}^{2}} & \lesssim\|h\|_{\ell^{2}} \varepsilon^{\frac{a}{2}+b-}, \\
\left\|Q_{a, b}^{h}\right\|_{\ell_{1 / 2-}^{2}} & \lesssim\|h\|_{\ell_{-1 / 2}^{2}} \varepsilon^{\frac{a}{2}+b-},
\end{aligned}
$$

and for any $b \geq 0$, we have

$$
\begin{aligned}
\left\|Q_{0, b}^{h}\right\|_{\ell^{2}} & \lesssim\|h\|_{\ell^{2}} \varepsilon^{b-}, \\
\left\|Q_{0, b}^{h}\right\|_{\ell_{-1 / 2}^{2}} & \lesssim\|h\|_{\ell_{-1 / 2}^{2}} \varepsilon^{b-} .
\end{aligned}
$$

We now prove Theorem 5.1 using Propositions 5.1, 5.2 and 5.3.

Proof of Theorem 5.1. By the discussion leading to (39) we need to estimate $H_{3}\left(Q_{a_{1}, b_{1}}^{h}, Q_{a_{2}, b_{2}}, Q_{a_{3}, b_{3}}\right)$ for $h \in \ell^{2}$ and $h \in \ell_{-1 / 2}^{2}$. First consider the case 
$h \in \ell^{2}$. We have the following subcases $a_{1}=0$, and $a_{1} \neq 0$. In the former case, by Proposition 5.1 and Proposition 5.3, we have

$$
\begin{aligned}
\left|H_{3}\left(Q_{0, b_{1}}^{h}, Q_{a_{2}, b_{2}}, Q_{a_{3}, b_{3}}\right)\right| & \lesssim\left\|Q_{0, b_{1}}^{h}\right\|_{\ell^{2}}\left\|Q_{a_{2}, b_{2}}\right\|_{\ell_{1+}^{2}}\left\|Q_{a_{3}, b_{3}}\right\|_{\ell_{1+}^{2}} \\
& \lesssim\|h\|_{\ell^{2}} \varepsilon^{b_{1}-}\left\|Q_{a_{2}, b_{2}}\right\|_{\ell_{1+}^{2}}\left\|Q_{a_{3}, b_{3}}\right\|_{\ell_{1+}^{2}} .
\end{aligned}
$$

Now, by Proposition 5.2, it is easy to see that the worst case is when $a_{2}=a$, $a_{3}=0$, in which case we obtain

$$
\begin{aligned}
\left|H_{3}\left(Q_{0, b_{1}}^{h}, Q_{a_{2}, b_{2}}, Q_{a_{3}, b_{3}}\right)\right| & \lesssim\|h\|_{\ell^{2}} \varepsilon^{b_{1}-} \varepsilon^{\frac{1}{2}+\frac{a}{2}+b_{2}-} \varepsilon^{-\frac{1}{2}+b_{3}-} \\
& \lesssim\|h\|_{\ell^{2}} \varepsilon^{\frac{a}{2}+b-} .
\end{aligned}
$$

If $a_{1} \neq 0$, the worst case is when $a_{1}=a, a_{2}=a_{3}=0$. Using the Propositions above we have

$$
\begin{aligned}
\left|H_{3}\left(Q_{a, b_{1}}^{h}, Q_{0, b_{2}}, Q_{0, b_{3}}\right)\right| & \lesssim\left\|Q_{a, b_{1}}^{h}\right\|_{\ell_{1-}^{2}}\left\|Q_{0, b_{2}}\right\|_{\ell_{1 / 2+}^{2}}\left\|Q_{0, b_{3}}\right\|_{\ell_{1 / 2+}^{2}} \\
& \lesssim\|h\|_{\ell^{2}} \varepsilon^{\frac{a}{2}+b_{1}-} \varepsilon^{b_{2}-} \varepsilon^{b_{3}-} \\
& \lesssim\|h\|_{\ell^{2}} \varepsilon^{\frac{a}{2}+b-} .
\end{aligned}
$$

It remains to consider the case $h \in \ell_{-1 / 2}^{2}$. As before we have the subcases $a_{1}=0, a_{1} \neq 0$. If $a_{1}=0$, the worst case is when $a_{2}=a, a_{3}=0$. We estimate

$$
\begin{aligned}
& \left|H_{3}\left(Q_{0, b_{1}}^{h}, Q_{a, b_{2}}, Q_{0, b_{3}}\right)\right| \\
& \lesssim\left\|Q_{0, b_{1}}^{h}\right\|_{\ell_{-1 / 2}^{2}}\left(\left\|Q_{a, b_{2}}\right\|_{\ell_{3 / 2}^{2}}\left\|Q_{0, b_{3}}\right\|_{\ell_{1+}^{2}}+\left\|Q_{a, b_{2}}\right\|_{\ell_{1+}^{2}}\left\|Q_{0, b_{3}}\right\|_{\ell_{3 / 2}^{2}}\right) \\
& \lesssim\|h\|_{\ell_{-1 / 2}^{2}} \varepsilon^{b_{1}-}\left(\varepsilon^{\frac{a}{2}+b_{2}-} \varepsilon^{-\frac{1}{2}+b_{3}-}+\varepsilon^{\frac{1}{2}+\frac{a}{2}+b_{2}-} \varepsilon^{-1+b_{3}-}\right) \\
& \lesssim\|h\|_{\ell_{-1 / 2}^{2}} \varepsilon^{-\frac{1}{2}+\frac{a}{2}+b-} .
\end{aligned}
$$

If $a_{1} \neq 0$, the worst case is when $a_{1}=a, a_{2}=a_{3}=0$. We estimate

$$
\begin{aligned}
\left|H_{3}\left(Q_{a, b_{1}}^{h}, Q_{0, b_{2}}, Q_{0, b_{3}}\right)\right| & \lesssim\left\|Q_{a, b_{1}}^{h}\right\|_{\ell_{1 / 2-}^{2}}\left\|Q_{0, b_{2}}\right\|_{\ell_{1}^{2}}\left\|Q_{0, b_{3}}\right\|_{\ell_{1 / 2+}^{2}} \\
& \lesssim\|h\|_{\ell_{-1 / 2}^{2}} \varepsilon^{\frac{a}{2}+b_{1}-} \varepsilon^{-\frac{1}{2}+b_{2}-} \varepsilon^{b_{3}-} \\
& \lesssim\|h\|_{\ell_{-1 / 2}^{2}} \varepsilon^{-\frac{1}{2}+\frac{a}{2}+b-}
\end{aligned}
$$

Now we prove Propositions 5.1, 5.2, 5.3.

Proof of Proposition 5.1. To prove the Proposition we will repeatedly use, without mentioning, the results of Lemma 4.3. Since $H_{3}$ is symmetric in $q_{1}, q_{2}, q_{3}$, it suffices to consider the case $\left(j_{1}, j_{2}, j_{3}\right)=(1,2,3)$. We start with 
the second assertion:

$$
\begin{aligned}
\left|H_{3}\left(q_{1}, q_{2}, q_{3}\right)\right| & \lesssim \sum_{n_{1}+n_{2}+n_{3}=0} \sqrt{n_{1} n_{2} n_{3}}\left|q_{1}\left(n_{1}\right) q_{2}\left(n_{2}\right) q_{3}\left(n_{3}\right)\right| \\
& =\left\langle\sqrt{\cdot}\left|q_{1}\right|, \sqrt{\cdot}\left|q_{2}\right| * \sqrt{\cdot}\left|q_{3}\right|\right\rangle \lesssim\left\|\sqrt{\cdot} q_{1}\right\|_{\ell^{1+}}\left\|\sqrt{\cdot}\left|q_{2}\right| * \sqrt{\cdot}\left|q_{3}\right|\right\|_{\ell^{\infty-}} \\
& \lesssim\left\|q_{1}\right\|_{\ell_{1-}^{2}}\left\|\sqrt{\cdot} q_{2}\right\|_{\ell^{2-}}\left\|\sqrt{\cdot} q_{3}\right\|_{\ell^{2-}} \lesssim\left\|q_{1}\right\|_{\ell_{1-}^{2}}\left\|q_{2}\right\|_{\ell_{1 / 2+}^{2}}\left\|q_{3}\right\|_{\ell_{1 / 2+}^{2}} .
\end{aligned}
$$

To prove the other three assertions note that for $s<1 / 2$,

$$
\begin{aligned}
& \left|H_{3}\left(q_{1}, q_{2}, q_{3}\right)\right| \lesssim \\
& \lesssim \sum_{n_{1}+n_{2}+n_{3}=0}\left|n_{1}\right|^{s}\left(\left|n_{2}\right|^{1-s}\left|n_{3}\right|^{1 / 2}+\left|n_{2}\right|^{1 / 2}\left|n_{3}\right|^{1-s}\right)\left|q_{1}\left(n_{1}\right) q_{2}\left(n_{2}\right) q_{3}\left(n_{3}\right)\right| \\
& =\left\langle|\cdot|^{s}\left|q_{1}\right|,\left.|\cdot|\right|^{1-s}\left|q_{2}\right| *|\cdot|{ }^{1 / 2}\left|q_{3}\right|\right\rangle+\left\langle|\cdot|^{s}\left|q_{1}\right|,|\cdot|{ }^{1 / 2}\left|q_{2}\right| *|\cdot|{ }^{1-s}\left|q_{3}\right|\right\rangle \\
& \quad \lesssim\left\|q_{1}\right\|_{\ell_{s}^{2}}\left(\left\|\left.|\cdot|\right|^{1-s}\left|q_{2}\right| *|\cdot|{ }^{1 / 2}\left|q_{3}\right|\right\|_{\ell^{2}}+\left\||\cdot|^{1 / 2}\left|q_{2}\right| *|\cdot|{ }^{1-s}\left|q_{3}\right|\right\|_{\ell^{2}}\right) .
\end{aligned}
$$

For $s=0$, we bound (40) by

$$
\left\|q_{1}\right\|_{\ell^{2}}\left(\left\|q_{2}\right\|_{\ell_{1}^{2}}\left\|q_{3}\right\|_{\ell_{1 / 2}^{1}}+\left\|q_{2}\right\|_{\ell_{1 / 2}^{1}}\left\|q_{3}\right\|_{\ell_{1}^{2}}\right) \lesssim\left\|q_{1}\right\|_{\ell^{2}}\left\|q_{2}\right\|_{\ell_{1+}^{2}}\left\|q_{3}\right\|_{\ell_{1+}^{2}}
$$

which proves the first assertion. For $s=-1 / 2$, we bound (40) by

$$
\begin{aligned}
\left\|q_{1}\right\|_{\ell_{-1 / 2}^{2}}\left(\left\|q_{2}\right\|_{\ell_{3 / 2}^{2}}\left\|q_{3}\right\|_{\ell_{1 / 2}^{1}}\right. & \left.+\left\|q_{2}\right\|_{\ell_{1 / 2}^{1}}\left\|q_{3}\right\|_{\ell_{3 / 2}^{2}}\right) \\
& \lesssim\left\|q_{1}\right\|_{\ell_{-1 / 2}^{2}}\left(\left\|q_{2}\right\|_{\ell_{3 / 2}^{2}}\left\|q_{3}\right\|_{\ell_{1+}^{2}}+\left\|q_{2}\right\|_{\ell_{1+}^{2}}\left\|q_{3}\right\|_{\ell_{3 / 2}^{2}}\right),
\end{aligned}
$$

which proves the third assertion. Finally for $s=1 / 2-\delta, \delta>0$, we bound (401) by

$$
\begin{aligned}
\left\|q_{1}\right\|_{\ell_{1 / 2-\delta}^{2}}\left(\left\|q_{2}\right\|_{\ell_{1 / 2+\delta}^{1+}}\left\|q_{3}\right\|_{\ell_{1 / 2}^{2-}}+\left\|q_{2}\right\|_{\ell_{1 / 2}^{1+}}\left\|q_{3}\right\|_{\ell_{1 / 2+\delta}^{2-}}\right) & \\
& \lesssim\left\|q_{1}\right\|_{\ell_{1 / 2-\delta}^{2}}\left(\left\|q_{2}\right\|_{\ell_{1}^{2}}\left\|q_{3}\right\|_{\ell_{1 / 2+}^{2}}+\left\|q_{2}\right\|_{\ell_{1}^{2}}\left\|q_{3}\right\|_{\ell_{1 / 2+\delta+}^{2}}\right) .
\end{aligned}
$$

Proof of Proposition 5.2. We start with the case $a=0$. Note that for $b=0$, the statement is true for any $s \in[0,3 / 2]$ since $Q_{0,0}=q$. For $b \geq 1$, we use a simple induction. We can write

$$
Q_{0, b}=f_{2}\left(Q_{0, b_{1}}, Q_{0, b_{2}}, Q_{0, b_{3}}\right),
$$

with $b_{1}+b_{2}+b_{3}=b-1$. Using Lemma 4.2, for any $s \in[0,3 / 2]$, we have

$$
\left\|Q_{0, b}\right\|_{\ell_{s}^{2}} \lesssim \sum\left\|Q_{0, b_{j_{1}}}\right\|_{\ell_{s}^{2}}\left\|Q_{0, b_{j_{2}}}\right\|_{\ell_{0+}^{2}}\left\|Q_{0, b_{j_{3}}}\right\|_{\ell_{0+}^{2}},
$$

where the sum is over all permutations $\left(j_{1}, j_{2}, j_{3}\right)$ of $(1,2,3)$. By the induction hypothesis the last sum can be estimated by

$$
\left\|Q_{0, b}\right\|_{\ell_{s}^{2}} \lesssim \sum \varepsilon^{\frac{1}{2}-s+b_{j_{1}}-} \varepsilon^{\frac{1}{2}+b_{j_{2}}-} \varepsilon^{\frac{1}{2}+b_{j_{3}}-} \lesssim \varepsilon^{\frac{1}{2}-s+b-} .
$$


In the case $a \geq 1$, we set up an induction on $a$. We first prove that the statement is valid for $a=1$ and for any $s \in[1,3 / 2], b \geq 0$. We write

$$
Q_{1, b}=f_{1}\left(Q_{0, b_{1}}, Q_{0, b_{2}}\right),
$$

$b_{1}+b_{2}=b$. By Lemma 4.1 we estimate

$$
\begin{aligned}
\left\|Q_{1, b}\right\|_{\ell_{3 / 2}^{2}} & =\left\|f_{1}\left(Q_{0, b_{1}}, Q_{0, b_{2}}\right)\right\|_{\ell_{3 / 2}^{2}} \lesssim\left\|Q_{0, b_{1}}\right\|_{\ell_{1 / 2}^{2}}\left\|Q_{0, b_{2}}\right\|_{\ell_{0+}^{2}}+\left\|Q_{0, b_{2}}\right\|_{\ell_{1 / 2}^{2}}\left\|Q_{0, b_{1}}\right\|_{\ell_{0+}^{2}} \\
& \lesssim \varepsilon^{b_{1}-} \varepsilon^{\frac{1}{2}+b_{2}-}+\varepsilon^{b_{2}-} \varepsilon^{\frac{1}{2}+b_{1}-} \lesssim \varepsilon^{\frac{1}{2}+b-} .
\end{aligned}
$$

Again by Lemma 4.1 we estimate

$\left\|Q_{1, b}\right\|_{\ell_{1-}^{2}}=\left\|f_{1}\left(Q_{0, b_{1}}, Q_{0, b_{2}}\right)\right\|_{\ell_{1-}^{2}} \lesssim\left\|Q_{0, b_{1}}\right\|_{\ell^{2}}\left\|Q_{0, b_{2}}\right\|_{\ell^{2}} \lesssim \varepsilon^{\frac{1}{2}+b_{1}-} \varepsilon^{\frac{1}{2}+b_{2}-} \lesssim \varepsilon^{1+b-}$.

Now by a simple interpolation, for $s \in[1,3 / 2)$,

$$
\left\|Q_{1, b}\right\|_{\ell_{s}^{2}} \lesssim\left\|Q_{1, b}\right\|_{\ell_{1-}^{2}}^{\theta}\left\|Q_{1, b}\right\|_{\ell_{3 / 2}^{2}}^{1-\theta} \lesssim \varepsilon^{\theta(1+b-)} \varepsilon^{(1-\theta)\left(\frac{1}{2}+b-\right)}=\varepsilon^{\frac{1+\theta}{2}+b-},
$$

where $\theta=3-2 s-$. This implies

$$
\left\|Q_{1, b}\right\|_{\ell_{s}^{2}} \lesssim \varepsilon^{2-s+b-}
$$

We proceed by induction on $a>1$. We have

$$
Q_{a, b}=f_{1}\left(Q_{a_{1}, b_{1}}, Q_{a_{2}, b_{2}}\right)
$$

$a_{1}+a_{2}=a-1, b_{1}+b_{2}=b$. The worst case (in terms of gain in powers of $\varepsilon)$ is when $a_{1}=a-1$ and $a_{2}=0$. As above, using the induction hypothesis and Lemma 4.1 we have

$$
\begin{aligned}
\left\|Q_{a, b}\right\|_{\ell_{3 / 2}^{2}} & \lesssim\left\|Q_{a-1, b_{1}}\right\|_{\ell_{1 / 2}^{2}}\left\|Q_{0, b_{2}}\right\|_{\ell_{0+}^{2}}+\left\|Q_{0, b_{2}}\right\|_{\ell_{1 / 2}^{2}}\left\|Q_{a-1, b_{1}}\right\|_{\ell_{0+}^{2}} \\
& \lesssim\left\|Q_{a-1, b_{1}}\right\|_{\ell_{1}^{2}}\left\|Q_{0, b_{2}}\right\|_{\ell_{1 / 2}^{2}} \lesssim \varepsilon^{\frac{a}{2}+b_{1}-} \varepsilon^{b_{2}-}=\varepsilon^{\frac{a}{2}+b-} .
\end{aligned}
$$

Similarly,

$$
\begin{aligned}
\left\|Q_{a, b}\right\|_{\ell_{1-}^{2}} & \lesssim\left\|Q_{a-1, b_{1}}\right\|_{\ell^{2}}\left\|Q_{0, b_{2}}\right\|_{\ell^{2}} \lesssim\left\|Q_{a-1, b_{1}}\right\|_{\ell_{1}^{2}}\left\|Q_{0, b_{2}}\right\|_{\ell^{2}} \\
& \lesssim \varepsilon^{\frac{a}{2}+b_{1}-} \varepsilon^{\frac{1}{2}+b_{2}-} \lesssim \varepsilon^{\frac{1}{2}+\frac{a}{2}+b-} .
\end{aligned}
$$

The statement for $s \in[1,3 / 2)$ follows from interpolation as above.

Proof of Proposition 5.3. We give a proof only for the case $h \in \ell_{-1 / 2}^{2}$. The proof for the case $h \in \ell^{2}$ is essentially the same. We start with the case $a=0$. Note that for $b=0$, the statement is true since $Q_{0,0}^{h}=h$. For $b \geq 1$, we use a simple induction. We can write without loss of generality

$$
Q_{0, b}^{h}=f_{2}\left(Q_{0, b_{1}}^{h}, Q_{0, b_{2}}, Q_{0, b_{3}}\right),
$$

with $b_{1}+b_{2}+b_{3}=b-1$. Using Lemma 4.2, we have

$$
\begin{aligned}
\left\|Q_{0, b}^{h}\right\|_{\ell_{-1 / 2}^{2}} & \lesssim\left\|Q_{0, b_{1}}^{h}\right\|_{\ell_{-1 / 2}^{2}}\left\|Q_{0, b_{2}}\right\|_{\ell_{0+}^{2}}\left\|Q_{0, b_{3}}\right\|_{\ell_{0+}^{2}} \\
& \lesssim\|h\|_{\ell_{-1 / 2}^{2}} \varepsilon^{b_{1}-} \varepsilon^{\frac{1}{2}+b_{2}-} \varepsilon^{\frac{1}{2}+b_{3}-}=\|h\|_{\ell_{-1 / 2}^{2}} \varepsilon^{b-} .
\end{aligned}
$$


The second inequality follows from the induction hypothesis and Proposition 5.2 .

In the case $a \geq 1$, we set up an induction on $a$. We first prove that the statement is valid for $a=1$ for any $b \geq 0$. We write, without loss of generality,

$$
Q_{1, b}^{h}=f_{1}\left(Q_{0, b_{1}}^{h}, Q_{0, b_{2}}\right),
$$

$b_{1}+b_{2}=b$. By Lemma 4.1 we estimate

$$
\begin{aligned}
\left\|Q_{1, b}^{h}\right\|_{\ell_{1 / 2-}^{2}} & =\left\|f_{1}\left(Q_{0, b_{1}}^{h}, Q_{0, b_{2}}\right)\right\|_{\ell_{1 / 2-}^{2}} \lesssim\left\|Q_{0, b_{1}}^{h}\right\|_{\ell_{-1 / 2}^{2}}\left\|Q_{0, b_{2}}\right\|_{\ell^{2}} \\
& \lesssim\|h\|_{\ell_{-1 / 2}^{2}} \varepsilon^{b_{1}-} \varepsilon^{\frac{1}{2}+b_{2}-} \lesssim\|h\|_{\ell_{-1 / 2}^{2}} \varepsilon^{\frac{1}{2}+b-} .
\end{aligned}
$$

The second inequality follows from the first part of the proof and Proposition 5.2 .

We proceed by induction on $a>1$. We have, without loss of generality,

$$
Q_{a, b}^{h}=f_{1}\left(Q_{a_{1}, b_{1}}^{h}, Q_{a_{2}, b_{2}}\right)
$$

$a_{1}+a_{2}=a-1, b_{1}+b_{2}=b$. Using Lemma 4.1, we have

$$
\begin{aligned}
\left\|Q_{a, b}^{h}\right\|_{\ell_{1 / 2-}^{2}} & \lesssim\left\|Q_{a_{1}, b_{1}}^{h}\right\|_{\ell_{-1 / 2}^{2}}\left\|Q_{a_{2}, b_{2}}\right\|_{\ell^{2}} \\
& \lesssim\|h\|_{\ell_{-1 / 2}^{2}} \varepsilon^{\frac{a_{1}}{2}+b_{1}-\varepsilon^{\frac{1}{2}+\frac{a_{2}}{2}+b_{2}-} \lesssim\|h\|_{\ell_{-1 / 2}^{2}} \varepsilon^{\frac{a}{2}+b-} .} .
\end{aligned}
$$

The second inequality follows from the induction hypothesis and Proposition 5.2 by considering the cases $a_{1}=0, a_{1} \neq 0$ and $a_{2}=0, a_{2} \neq 0$.

5.2. Remainder estimates. In this section we estimate the error terms involving integrals. By (20) and (25), it suffices to prove the following inequalities

$$
\begin{array}{cr}
\sup _{\tau \in[0,1]}\left\|\partial_{q}\left(g_{F_{1}}^{3} H_{3} \circ \Phi_{F_{1}}^{\tau}\right)\right\|_{\ell_{s}^{2}} \lesssim \varepsilon^{1-s-}, \quad s \in[0,1 / 2] \\
\sup _{\tau \in[0,1]}\left\|\partial_{q} g_{F_{2}}\left(g_{F_{1}}^{3} H_{3} \circ \Phi_{F_{1}}^{\tau}\right)\right\|_{\ell_{s}^{2}} \lesssim \varepsilon^{1-s-}, & s \in[0,1 / 2] \\
\sup _{\tau \in[0,1]}\left\|\partial_{q}\left(g_{F_{2}}^{2}\left(H \circ \Phi_{F_{1}}^{1}\right) \circ \Phi_{F_{2}}^{\tau}\right)\right\|_{\ell_{s}^{2}} \lesssim \varepsilon^{1-s-}, & s \in[0,1 / 2] .
\end{array}
$$

To prove (41), let $w=\Phi_{F_{1}}^{\tau}(q)$. Note that

$$
\begin{aligned}
\left\|\partial_{q}\left(g_{F_{1}}^{a} H_{3} \circ \Phi_{F_{1}}^{\tau}\right)\right\|_{\ell_{s}^{2}} & =\sup _{\|h\|_{\ell_{-s}^{2}}=1}\left|\sum_{m, k} \frac{\partial g_{F_{1}}^{a} H_{3}}{\partial w(m)} \frac{\partial w(m)}{\partial q(-k)} h(k)\right| \\
& =\sup _{\|h\|_{\ell_{-s}^{2}}=1}\left|\sum_{m} \frac{\partial g_{F_{1}}^{a} H_{3}}{\partial w(m)}\left(\sum_{k} \frac{\partial w(m)}{\partial q(-k)} h(k)\right)\right| \\
& \lesssim\left\|\partial_{w} g_{F_{1}}^{a} H_{3}\right\|_{\ell_{s}^{2}} \sup _{\|h\|_{\ell_{-s}^{2}}}\left\|\sum_{k} \frac{\partial w(m)}{\partial q(-k)} h(k)\right\|_{\ell_{-s}^{2}} .
\end{aligned}
$$

Since $F_{1}$ is near identity, by our assumptions on $q,\|w\|_{\ell_{s}^{2}} \lesssim \varepsilon^{\frac{1}{2}-s}$ for $s \in$ $[0,3 / 2]$. Therefore, Theorem 5.1 implies that $\left\|\partial_{w} g_{F_{1}}^{a} H_{3}\right\|_{\ell_{s}^{2}} \lesssim \varepsilon^{1-s-}$ (for 
$a \geq 2)$. Thus, it suffices to prove that

$$
\|T(h)\|_{\ell_{-s}^{2}} \lesssim\|h\|_{\ell_{-s}^{2}}, \quad s \in[0,1 / 2]
$$

where $T(h):=\sum_{k} D_{k} w(m) h(k)$, and $D_{k} w(m):=\frac{\partial w(m)}{\partial q(-k)}$.

To prove (44) first note that $w(m)$ is the solution at $t=\tau$ of the system

$$
\frac{d w(m)}{d t}=\frac{\partial F_{1}}{\partial w(-m)}=f_{1}(w, w)(m),\left.\quad w\right|_{t=0}=q .
$$

Differentiating this equation with respect to initial condition $q(-k)$, we see that

$$
\frac{d D_{k} w(m)}{d t}=2 f_{1}\left(D_{k} w, w\right),\left.\quad D_{k} w(m)\right|_{t=0}=\delta_{-k, m} .
$$

Pairing both sides with $h(k)$, we have the following equation for $T(h)$

$$
\frac{d T(h)(m)}{d t}=2 f_{1}(T(h), w),\left.\quad T(h)(m)\right|_{t=0}=h(-m) .
$$

Therefore (44) is satisfied at $\tau=0$, and by Gronwall's lemma (since $\tau \in$ $[0,1])$, it suffices to see that $\left\|f_{1}(T(h), w)\right\|_{\ell_{-s}^{2}} \lesssim\|T(h)\|_{\ell_{-s}^{2}}$ for $s \in[0,1 / 2]$.

This immediately follows from Lemma 4.1.

The remaining estimates (42) and (43) follow from similar considerations using Lemma 4.1, Lemma 4.2, and Theorem 5.1, We omit the details.

\section{REFERENCES}

[1] J. Bourgain,Fourier transform restriction phenomena for certain lattice subsets and applications to nonlinear evolution equations. Part I: Schrödinger equations, GAFA, 3 No. 2 (1993), 107-156.

[2] J. Bourgain, Fourier transform restriction phenomena for certain lattice subsets and applications to nonlinear evolution equations. Part II: The KdV equation, GAFA, 3 (1993), 209-262.

[3] J. Colliander, M. Keel, G. Staffilani, H. Takaoka, T. Tao, Weakly turbulent solutions for the cubic defocusing nonlinear Schrdinger equation, preprint, http://arxiv.org/abs/0808.1742.

[4] M. B. Erdoğan, V. Zharnitsky, Quasi-linear dynamics in nonlinear Schrödinger equation with periodic boundary conditions, Commun. Math. Phys. 281 (2008), 655-673.

[5] N. Hayashi, P. Naumkin, Asymptotics for large time of solutions to the nonlinear Schrödinger and Hartree equations, Amer. Jour. Math. 120 (1998), 369-389.

[6] T. Kapeller, J. Pöschel, $K d V$ and $K A M$, A Series of Modern Surveys in Mathematics 45, Springer-Verlag, Berlin, (2003).

[7] C. Kharif, E. Pelinovsky, Physical mechanisms of rogue wave phenomenon, Eur. Jour. Mech. B/Fluids, 22 (2003) 603-634.

[8] S. Kuksin, Analysis of Hamiltonian PDEs, Oxford University Press, New York, 2000.

[9] R. Temam, Sur un probleme non lineaire, J. Math. Pures Appl. 48 (1969), 159-172.

Department of Mathematics, University of Illinois, Urbana, IL 61801, U.S.A.

E-mail address: berdogan@uiuc.edu, tzirakis@math.uiuc.edu, vzh@uiuc.edu 